\title{
3 Schulhäuser als Orte pädagogisch initiierter Sozialisationsprozesse - Theoretische Annäherung und Ableitung der Fragestellung
}

Das folgende Kapitel dient der Annäherung an den Forschungsgegenstand Schulhaus aus einer architektur- und bildungstheoretischen sowie einer methodologischen Perspektive, die konstitutionstheoretische Voraussetzungen enthält. Das Ziel ist die Ableitung und Klärung der Fragestellung über die Ausformulierung der theoretischen Annahmen, die für das Verständnis des Untersuchungsgegenstandes bestimmend sind. Zu klären ist, was die Architektur als Untersuchungs- und Analysegegenstand an Ergebnissen verspricht, was nicht über andere, zugänglichere und erprobte Daten erreicht werden könnte. Denn das Forschungsprojekt ist wesentlich als ein architektursoziologisches Unterfangen konzipiert, und das Ziel dieser Annäherung ist es, den Gegenstand Architektur als sozialwissenschaftliches Datum konturiert herauszuarbeiten. Den theoretischen Ausführungen sind einige Bemerkungen zum Untersuchungsgegenstand und der Forschungsstand vorangestellt.

\subsection{Raum- und architektursoziologische Überlegungen zur gebauten Bildung}

Da das Forschungsprojekt wesentlich als ein architektursoziologisches Unterfangen konzipiert ist, wird eine Theorie benötigt, welche die vielseitigen Wechselwirkungen und Wirkmächtigkeiten von Architektur fassen kann und diese als soziologische Theorie aus dem Sozialen herleitet. Zudem muss sie konstitutionstheoretisch und methodologisch die Erschließung der in Architektur geronnenen Sinn- und Bedeutungsstrukturen explizieren können und zugleich den formativpraxisleitenden Charakter von Architektur erklären können. Es ist als Erstes zu klären, welches theoretische Gerüst oder Paradigma versprechen könnte, den Ansatz zu verfolgen. Steets (2015: 19 ff.) unterscheidet drei wesentliche unterschiedliche Perspektiven, die die architektursoziologische Diskussion prägen. Sie sollen hier stark verkürzt wiedergegeben werden, um das Forschungsvorhaben darin zu verorten. 
Als Erstes unterscheidet Steets eine strukturtheoretische Position, bei der die Architektur ,als materialisierte Strukturen des Sozialen“ (Steets 2015: 19 ff.) betrachtet werden kann. In dieser Perspektive ist die Denkweise dominant, dass Dinge und Gebäude als „materielles Substrat des Gesellschaftlichen“ (Durkheim 1961) wahrgenommen werden, die damit zu einem „Anzeiger des Sozialen“ (Elias 2003) werden. Es geht - stark verkürzt - darum, Architektur als das zu lesen, was sie repräsentiert. Dabei wird in dieser Perspektive die stabilisierende Funktion der Architektur auf die soziale Ordnung stark gewichtet. Durch die Dauerhaftigkeit von Architekturen amtieren diese als „kollektives Gedächtnis“ (Halbwachs 1997) einer Gesellschaft.

Eine zweite Perspektive fokussiert darauf, wie Menschen mit Gebäuden umgehen. Die Bedeutung von Dingwelt und Architektur wird nicht als materiales Substrat in den Dingen gesehen, sondern sie ist eine Folge davon, wie Menschen mit Dingen interagieren. Die Bedeutung und den Sinn z. B. eines Schulhauses erhält dieses erst durch die Praktiken mit dem Haus und in dem Haus. Der Umgang mit Dingen wird im Wesentlichen darüber bestimmt, dass der Mensch Kontrolle über sich selbst und über die Umwelt ausübt (zum Umgang mit Dingen siehe Mead 1998: 296 ff.). Diese Perspektive betrachtet Dinge und Gebäude primär unter dem Aspekt, welche symbolischen Zuschreibungen (beispielsweise Bourdieu 1976, 2011) sie haben und mit welchen Praktiken sie verbunden werden.

In einer dritten Perspektive fasst Steets Sichtweisen zusammen, die „das Gebaute in den Mittelpunkt der Analyse [stellt] und (...) ihr explizit eine soziale Effektivität zu[-schreibt]“ (2015: 44). Es geht bei den Positionen im Wesentlichen darum, ,den greifbaren Unterschied, den Gebäude im Sozialen hinterlassen, als historisch kontingent zu dechiffrieren, und zweitens, das Statische, das gebaute Strukturen per se anzuhaften scheint, ,in Bewegung" zu versetzen“ (ebd.; Hervorhebungen im Original). Steets verweist vor allem auf den diskursanalytischen Untersuchungen Foucaults und auf die Actor-network theory (ANT) von Latour (Latour \& Yaneva 2008). Gemeinsamkeit ist, dass Dingen eine Intention zugeschrieben ist; sie haben ein Handlungsprogramm. Im deutschsprachigen Raum sind es vor allem die Architektursoziologien von Delitz (2009, 2010), Steets (2015) und von Fischer (2009, 2017), die diesen Anspruch verfolgen. 
Delitz (2010) leitet ihre Theorie der „gebauten Gesellschaft“ aus einer Perspektive vom Kollektiven her. Architektur realisiert sich aus einem vorerst imaginären Gesellschaftsentwurf, der imaginäre soziale Institutionen darstellt. Diese werden von den seelisch und psychisch instabilen Subjekten als Außenhalt und handlungsformende Gestalt angenommen. Architektur stellt damit ein ,Medium “ oder eine Vermittlungsinstanz dar, das der Gesellschaft ein ,Gesicht ' gibt, womit sie sich erst erkennen kann.

Einen fast konträren Weg in einem annähernd analogen Dreischritt geht Steets (2015), die von der sinnlichen Wahrnehmung von Architektur beim Individuum ausgeht. Architektur sieht sie als ,Externalisierung' von Sozialem Sinn, der sich in Form von Gebäuden ,objektiviert" und von den Bewohner_innen vor allem dann ,internalisiert" wird, wenn diese einen produktiven Umgang damit finden können.

Einen dritten Weg geht Fischer (2009, 2017), der Architektur als ,Schweres Kommunikationsmedium" definiert, das sich durch eine eigenlogische Materialität auszeichnet, die insbesondere durch ihre Omnipräsenz einen Konstitutionscharakter für das Soziale aufweist. Durch Architektur bzw. durch die Materialisierung der ,Exzentrischen Positionalität‘ erfahren Subjekte leiblich-körperlich die Differenz von System und Umwelt bzw. von Innen und Außen.

Der hauptsächliche Unterschied der vorliegenden Arbeit zu den Architektursoziologien von Fischer, Delitz und Steets liegt darin, dass das Ziel nicht eine philosophisch-anthropologische Begründung einer Architektursoziologie ist, sondern in erster Linie eine Programmatik für eine empirische Architektursoziologie darstellt. Das vorliegende Forschungsinteresse hat den Anspruch, die objektiv gegebenen Sinn- und Bedeutungsstrukturen zu rekonstruieren. Sie liegen in Form der Architektur metaphorisch gesagt, in Stein gemeißelt' dauerhaft vor. In diesem Sinne nimmt die Forschungsanlage Teile der strukturtheoretischen Positionen auf. Das strukturtheoretische Modell von sozialer Zeit und sozialem Raum nach Oevermann $(1995,2016)$ liefert dazu eine Theorie, welche Architektur als Sesshaftigkeit, als „Verankerung einer vergemeinschafteten Form von Sozialität“ (Oevermann 2003: 363) fasst. Mit dieser Konzeption kann Architektur nicht nur als Artefakt, sondern als objektivierte Positionalität einer kollektivaggregierten Lebenspraxis - z. B. der Schule - analysiert werden. Mit der Theorie der sozialen Raum-Zeitlichkeit wird zudem das Verhältnis von Raum und Architektur so geklärt, dass Architektur in seiner Dynamik zwischen Krise und 
Routine erfasst werden kann: konstitutionstheoretisch als krisenhafte Herstellung der Innen-Außen-Abgrenzung einer Lebenspraxis und lebenspraktisch als in Stein gegossene, auf Dauer gestellte Routinen, welche die Welt lesbar machen, soziale Strukturen stabilisieren und als objektivierte Regeln als Einflussstruktur für die Individuen handlungsleitend und -entlastend wirken.

Damit ist ein Element angesprochen, das die handlungslogische Komponente bestärkt, die der strukturalen Soziologie als handlungslogische Strukturtheorie inhärent ist. Struktur wird nicht als relationale Anordnung von Elementen verstanden, sondern als Eigengesetzlichkeit eines Falls. Gefasst als individuierte, lebensgeschichtlich motivierte Fallstruktur, kann sie sich zukunftsoffen jederzeit reproduzieren oder transformieren. Dies gilt auch für Gebäude, die als dauerhafte geplante Disposition einer sozialen Praxis für eine lange Zeit gebaut sind. Die Architektur eines Schulhauses gerät dann in eine Krise, wenn das Raumprogramm oder das Programm der Räume nicht mehr den Anforderungen zeitgemäßer Unterrichtsmethoden genügt oder auch wenn etwa demographische Entwicklungen einen Erweiterungs- oder Neubau verlangen.

Strukturtransformationen sind für das Forschungsvorhaben in einem doppelten Sinne bedeutsam. Nicht nur die Strukturtransformationen der Schulgebäude sind thematisch. Der Begriff Schulhaus bringt bereits wörtlich auf den Punkt, dass jedes Haus einen ,Auftrag ' hat, wofür es gebaut ist. Schulhäuser beherbergen Schulen. Sie sind gebaut, um das Kerngeschäft von Schulen, die gesellschaftlich organisierte und pädagogisch intendierte Bildung und Sozialisation, zu beherbergen und zu unterstützen. Deshalb wird Schulhäusern eine performative Wirkung zugeschrieben. Denn Bildungsprozesse sind Strukturtransformationen per se. Damit ist es der Auftrag von Schulbauten, Bildungs- und Sozialisationsprozesse zu unterstützen oder unter Umständen sogar zu initiieren, wie dies der zeitdiagnostische Diskurs von Schulhäusern als Lernumgebungen, Lern- und Bildungsräume und andere bedeutungsaffine Begriffe fordern.

Architektursoziologisch gesehen hat das Gebäude damit ein Handlungsprogramm, wie dies die Actor-network theory (ANT) postuliert (Latour 1999; Latour \& Yaneva 2008). Architekturen sind äußere Gegenstände, die auf Sozialisations- und Bildungsprozesse einwirken beziehungsweise diese mit ganz spezifischen Dispositionen leiten. Architekturen stellen Dinge zur Verfügung, die zu autonomer Aneignung einladen oder gewisse Praktiken unterbinden. Die handlungsleitenden Dispositionen von Architektur können mit der Theorie und 
Methodologie der Objektiven Hermeneutik jedoch gefasst werden, ohne dass den Dingen eine eigene Subjektivität zugeschrieben wird, wie dies bei der ANT der Fall ist (vgl. z. B. Callon 1986; Latour 1996).

Um den ,Auftrag ' der Gebäude zu fassen, wird auch ein bildungs- und sozialisationstheoretischer Zugang benötigt, welcher die Grundstruktur der Problematik von Schulraum als spezifisch gestaltetem Ort von pädagogischem Handeln beleuchtet. Besonders gut geeignet sind dazu die strukturtheoretischhandlungslogischen Sozialisations- und Bildungstheorien, da diese in der Unterscheidung von Krise und Routine Bildungs- und Lernprozesse präzise unterscheiden. In ihnen wird aber auch „die Differenz zwischen naturwüchsigen und professionellen Bildungskonstellationen präzise herausgearbeitet“ (Helsper 2012: 453).

In einem ersten Teil werden im nachfolgenden Kapitel die konstitutionstheoretischen Begriffe der Lebenspraxis in der Dynamik von Krise und Routine geklärt. In diese Dynamik eingelagert ist auch die sozialräumlich und sozialzeitlich leibliche Positionalität, die für den Gegenstand Architektur wesentlich ist. Daraus ableitend können die sozialen Funktionen und Wirkungen und insbesondere die ästhetischen Komponenten von Architektur diskutiert werden.

Der zweite Teil befasst sich mit dem Auftrag des Gebäudes, also mit der Schule als Bereich gesellschaftlich organisierter Bildung und Sozialisation. Schule soll in ihrer Struktur als Ort gesellschaftlich geplanter Bildungsveranstaltungen und -konstellationen herausgearbeitet werden, was auch die Differenz zwischen naturwüchsig informellen Bildungsorten und -situationen thematisch werden lässt. Im letzten Teil des zweiten Kapitels werden die beiden Perspektiven am Gegenstand Schulhaus zueinander in Bezug gesetzt, um daraus die Fragestellungen für das empirische Material abzuleiten. Wie kann Architektur als Repräsentation von Schule gelesen werden? Welche Erkenntnisse können durch die Analyse der Architektur von Schulhäusern über das Verhältnis von Schule und Gesellschaft gewonnen werden? Und wie erzeugt die Architektur eines Schulhauses eine suggestive und praxisleitende Wirkung für die Institution Schule?

Architektur ist eine komplexe Angelegenheit, wie Delitz ausführt: „Sie ist Kunst, Wissenschaft, Technik, Sozialtechnik, Artefakt; sie hat einen ökonomischen, juridischen, hygienischen, technologischen, einen Klassen-, Geschlechts- 
und Generationen-Aspekt“ (2009: 74). Diese Aufzählung könnte durch weitere Merkmale ergänzt werden.

Die vorliegende Arbeit verfolgt einen struktur- und handlungstheoretischen Zugang. Damit ist einerseits das methodische Vorgehen vorgezeichnet. In einer strukturalen Herangehensweise wird zuerst das zugrunde liegende Handlungsproblem eruiert, bevor an verschiedenen Schulbauten rekonstruiert wird, wie sie sich in ihrer Besonderheit als Lösungen des Handlungsproblems realisieren. Anderseits wird Architektur als Sesshaftigkeit, als Sitz und Verkörperung einer kollektiven Lebenspraxis betrachtet. Zudem sind Schulhäuser dafür erbaut, auf die Bildungsprozesse der Subjekte einzuwirken. Um dies konstitutionstheoretisch zu klären, bietet sich das Modell von Oevermann $(1995,2016)$ zur sozialen Zeit und zum sozialen Raum an. Mit diesen Überlegungen ist ein genuin soziologisches Modell vorhanden, das weder Aussagen über nichtsoziologische Geltungsbereiche macht, noch auf solche zurückgreift (Schmidtke 2006: 43). Architektur ist konstitutionstheoretisch fest mit dem sozialen Sein verwoben und nicht physisches Anhängsel oder passives Objekt. ${ }^{4}$

\subsubsection{Zur Strukturproblematik von Lebenspraxis}

Wesentlich für die strukturale Soziologie ist der Begriff der Lebenspraxis. Oevermann wählt den Begriff Lebenspraxis,

„,da er abstrakt und allgemein genug dafür ist, die Einheit des Lebendigen sowohl abgehoben vom Aggregierungsniveau der individuellen Person verallgemeinert zu erfassen als auch als einen fallstrukturgesetzlichen Zusammenhang von Soma, Psyche und Sozialität." (Oevermann 2004: 158)

Lebenspraxis bezieht sich nicht nur auf Personen, sondern bezeichnet auch ein Kollektiv: eine Familie, eine Dorfgemeinschaft, eine Stadt oder eine Nation. Lebenspraxen sind Einheiten des Lebendigen, die unterschiedlich aggregiert sind. Auch die Schule oder eine Gemeinde kann als eigenlogisch agierende

4 Für einen Überblick über die Diskussionen des oft unklaren Verhältnisses zwischen Architektur und Sozialem in den soziologischen Theorien siehe Delitz (2009: 74 ff.). 
Lebenspraxis erfasst werden. Denn die Struktur des Lebendigen konstituiert sich dadurch, dass sie um eine Lebensmitte zentriert ist und sich eine

„unbewusste zentrierte Subjektivität in ihrer Autonomie genau dadurch konstituiert, dass sie zugleich unter Entscheidungszwang steht, d. h. in einer Zukunftsoffenheit von Entscheidungsalternanten auswählen muss, und diese Entscheidung begründen können muss, obwohl eine echte Entscheidungssituation nur dann gegeben ist, wenn das Richtig-Falsch-Kalkül einer Begründung im selben Moment nicht erfüllt werden kann“ (Oevermann 2004: 159).

Damit ist der Kern angesprochen, in welchem sich die Lebenspraxis als autonomes handlungsfähiges Subjekt realisiert: in der selbsttätigen Bewältigung von Krisen.

Lebenspraxis konstituiert sich in Krisen (die folgenden Ausführungen basieren maßgeblich auf Garz \& Raven 2015; Oevermann 2000a, 2009a, 2016). Krisen sind Entscheidungssituationen, in der objektiv mehrere durch Regeln eröffnete Handlungsmöglichkeiten gegeben sind. Ein weiteres zentrales Merkmal von Krisen ist, dass sie einem nicht stillstehenden Handlungs- beziehungsweise Entscheidungsdruck unterliegen: Krisen müssen entschieden werden und können nicht aufgeschoben und schon gar nicht nicht entschieden werden. Der Gegenstand einer Krise ist für das Subjekt unbestimmt. Daher zeichnet sich eine Krise dadurch aus, dass keine Kriterien vorhanden sind, die den Entscheid im Voraus strukturieren. Die Wahl muss zwischen indifferenten Möglichkeiten getroffen werden, die nicht schon im Voraus durch Richtig-Falsch-Kalküle, Kosten-Nutzen-Kalküle, Präferenzen oder andere Kriterien begründet werden können. Andernfalls stellt die Entscheidungssituation keine echte Krise dar, da ein echtes Entscheidungsmoment nicht gegeben ist. Dass keine Begründungsmomente für die Auswahl vorhanden sind, bedeutet nicht, dass die Begründung aufgehoben ist, sondern vielmehr, dass sie erst nachträglich erbracht werden kann und erbracht werden muss, im Sinne einer nachträglichen reflexiven Bedeutungsgenerierung und Internalisierung in die Identität eines Subjektes. Daher sind Bildungsprozesse per se krisenhaft. Es sind Strukturtransformationsprozesse, in denen sich aus erfolgreich bewährten Krisen die erfolgreichen Wissensgehalte oder Fertigkeiten routinisieren.

Der Ablauf einer Krise kann mit einer Darstellung nach Oevermann (2016: 65) so modelliert werden: 


\begin{tabular}{|ccc|}
\hline S (-1) & S $(0)$ & S (+1) \\
Vollzug von Wirklichkeit $\rightarrow$ & eröffnete Möglichkeiten & \\
& (Parameter I) \\
& $\downarrow$ \\
& Vollzug von Wirklichkeit & \\
& (Parameter II) & $\rightarrow$ eröffnete Möglichkeiten \\
\hline
\end{tabular}

Abb. 1: Modell der Sequenzialität von Krisen

S (0) steht für die betrachtete Sequenz, an der gehandelt beziehungsweise die rekonstruiert wird. Lebenspraktisch stellt es die Gegenwärtigkeit im Hier und Jetzt dar, während $\mathrm{S}(-1)$ für die Vergangenheit und $\mathrm{S}(+1)$ für die Zukunft steht. Von links nach rechts liest sich also die temporale Abfolge der Sequenz. Von oben nach unten gelesen, beschreibt das Modell die nichttriviale Sequentiertheit der Lebenspraxis. Nichttrivial ist diese, da sie die Sequenzialität in einen Bedeutungszusammenhang stellt und einen regelgeleiteten sinnstrukturierten Zusammenhang von Handlungen herstellt. An der Stelle S (0) befindet sich der Erzeugungsparameter, auch Parameter I genannt. In Parameter I werden die wohlgeformten Handlungsmöglichkeiten aus ,algorithmisch operierenden, bedeutungsgenerierenden Regeln“ (Oevermann 2000a: 64) erzeugt. Dies sind Regeln der syntaktischen, semantischen, pragmatischen und grammatikalischen Regeln der Sprache. Dazu gehören aber auch die Regeln der Moral, der Sozialität, des logischen Schließens u. a. m.

Neben den universellen Regeln gibt es Regeln unterschiedlicher Reichweite, die historisch-kulturell in einer Epoche, für bestimmte Gruppen, z. B. Schüler_innen, oder in einem bestimmten Schulhaus oder einer Klasse gelten. Maiwald weist darauf hin, dass diese Regeln als soziale Konstruktionen betrachtet werden können. Es sind Regeln, die eine epistemische Objektivität insofern erlauben, als es Regeln oder ,,allgemein gültige konstitutive Regeln sind, die sozialen Tatsachen zugrunde liegen, die ihren ,institutionellen' Charakter begründen“ (Maiwald 2013: 190, Hervorhebung im Original). Am Beispiel einer Begrüßungsprozedur kann dies verdeutlicht werden. In der Situation, in der zwei Personen sich begegnen, sind Möglichkeiten gegeben: die des Grüßens und damit des Eröffnens einer gemeinsamen Praxis oder die des Nichtgrüßens. Indem die Person die andere begrüßt, wird autonome Lebenspraxis in der Selektion einer der objektiv gegebenen Möglichkeiten vollzogen. Die Begrüßung durch die erste Person eröffnet ihrerseits an der nächsten Sequenzstelle die beiden 
Möglichkeiten des Zurückgrüßens oder der Verweigerung des Grußes. Auf die Architektur übertragen fungieren Türen oder Umfriedungen als Eröffnungsprozeduren: Ich kann vor dem Schulhaus stehen, die Schranke der Umfriedung durchschreiten und den Schulhof betreten. Alternativ kann ich das Schulgelände links liegen lassen. Die Architektur hat den Charakter von Regeln, da sie Bedeutung erzeugt: zum Beispiel durch die Lage von Türen und Einfriedungen oder durch ihre Gesten. Man denke nur an die unterschiedlichen ,Begrüßungen“ durch die Eingangsbereiche von Hotels oder Gefängnissen.

Der Vollzug autonomer Lebenspraxis realisiert sich durch die Auswahl einer der eröffneten Möglichkeiten. Dieser wird im Modell als Parameter II oder Auswahlparameter bezeichnet. In Parameter II realisiert sich die faktisch getroffene Auswahl aus den eröffneten Möglichkeiten, und damit vollzieht sich Lebenspraxis in der Unmittelbarkeit der Gegenwärtigkeit. Das Modell von Lebenspraxis stellt eine nicht-stillstehende Ablaufkette von Krisen dar, da sich mit der getroffenen Auswahl neue Anschlussmöglichkeiten an der S (1) konstituieren, die nun in einer nächsten Betrachtung wiederum zu S (0) wird.

Aus erfolgreich gelösten Krisen emergieren Erfahrungen, die sich als Erfahrungswissen routinisieren und internalisiert werden. Sie liegen nun als Routinen abgespeichert vor, die in Zukunft für ähnlich oder gleich gelagerte Krisensituationen abgerufen werden und daher handlungsleitend sind. In ihrer Summe machen sie die Fallstrukturgesetzlichkeit einer Lebenspraxis aus: ihr Habitus in Form von Wahrnehmungs-, Deutungs- und Handlungsschemata, ihre Vorlieben, aber auch Neurosen usw. Die getroffenen Entscheidungen einer Lebenspraxis sind nie zufällig. Sie beruhen auf routinisierten Mustern, die die Fallstruktur in ihrer Eigengesetzlichkeit ausmachen.

Als konstitutionstheoretisches Modell von Lebenspraxis ist die Krise der Normalfall und die Routine die Ausnahme. Dieser Umstand ist forschungspraktisch für die Rekonstruktion zentral (vgl. dazu Kap. 4). Lebenspraktisch ist das Verhältnis von Krise und Routine umgekehrt, Routinen sind der Normalfall und Krisen der Grenzfall von Alltagsentscheidungen: Ich muss nicht jedes Mal von Neuem die Entscheidung treffen, ob ich den Schulhof überquere. Ich habe die Routine verinnerlicht, dass sich das nicht gehört, und nehme daher den Umweg außen herum in Kauf. Im Alltag sind es also äußere Normen - ,Das gehört sich nicht ${ }^{\star}$ - und innere Dispositionen, die als Routinen in Form von Wissen, Deutungsmustern, sozialen Normen, etwa durch Architektur, aber auch durch innere 
Motivationen des konkreten Subjektes, die Entscheidungen im Auswahlparameter vor- oder mitstrukturieren.

Die spezifische Selektion der bedeutungsgeladenen Möglichkeiten wird als Sinnstruktur der jeweils individuierten Lebenspraxis bezeichnet. Auch wenn Routinen alltagspraktisch den Normalfall darstellen, bedeutet das nicht, dass sie nicht jederzeit ihren ursprünglichen Krisencharakter wiedererlangen können und dies immer wieder auch tun. Ich kann mich beispielsweise nach Jahren einer bestimmten Routine dafür entscheiden, doch den Weg über den Schulhof zu nehmen. Oder als anderes Beispiel: Ein bewährtes Schulhaus kann in seiner Architektur in eine Krise geraten, da es aufgrund neuer pädagogischer Konzepte als nicht mehr adäquat gesehen wird, da die neuen Formen des Unterrichtens nach neuen Formen des architektonischen Raums verlangen. So ist es für jede Lebenspraxis normal, dass zwischen Strukturtransformationen, die immer in Krisen vonstattengehen, längere Phasen der Reproduktion der Strukturgesetzlichkeit eingelagert sind, in denen sich die Routinen bewähren.

\subsubsection{Soziale Raum-Zeitlichkeit-Architektur als Externalisierung von Identität}

Das Modell von Krise ist in eine temporale Abfolge eingebunden. Die Lebenspraxis vollzieht sich krisenhaft ausschließlich in der Gegenwärtigkeit. Lebenspraxis realisiert sich zwangsläufig auch räumlich: Sie vollzieht sich an einer Position im Hier. Dem Hier gegenüber steht das Dort, welches nur gedankenexperimentell vorhanden ist, also per se imaginiert ist. Dies geschieht zwingend entweder im Rückgriff auf die Vergangenheit: „Ich war dort“; oder mit Vorgriff auf die Zukunft: „Ich werde dort sein.“ In der Gegenwärtigkeit kann man nicht dort sein. Das bedeutet, dass die zeitliche und räumliche Trennung in der Gegenwärtigkeit des Krisenvollzugs untrennbar zusammenfällt. Oevermann (2016) spricht von der Positionalität, in der unmittelbaren krisenhaften Gegenwärtigkeit, in der Raum und Zeit ungeschieden sind. Raum und Zeit können ausschließlich im Modus der Routinen, also der sprachlich vermittelten Reflexion in der Nicht-Gegenwärtigkeit, getrennt betrachtet werden. Betrachten wir Raum und Zeit als voneinander Getrennte im Modell nach Oevermann (2016: 71). 


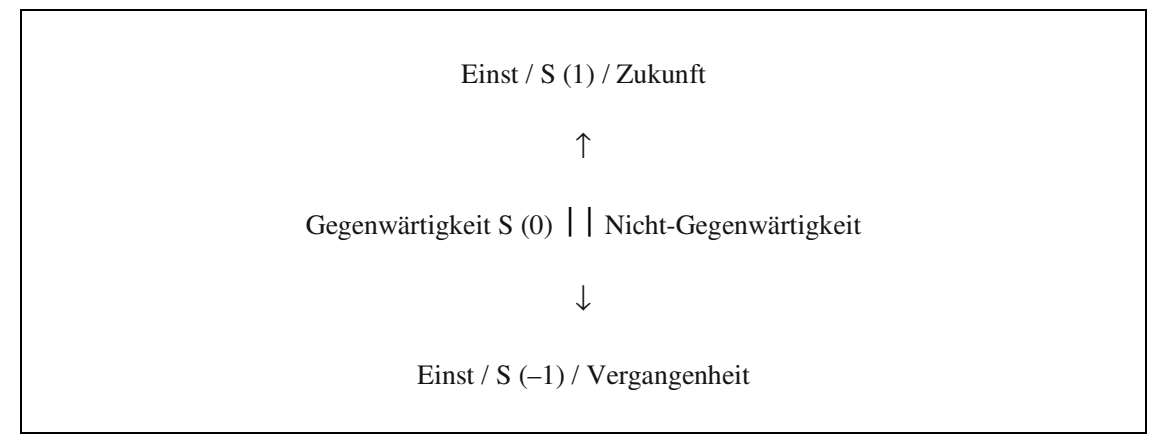

Abb. 2: Hierarchisch doppelte Opposition von Temporalität (Oevermann 2016: 71)

In zeitlicher Hinsicht scheidet sich die Gegenwärtigkeit im Jetzt an der Stelle S (0) von der Nicht-Gegenwärtigkeit durch das Einst an den Sequenzstellen S (-1) und S (1). Dabei wird ersichtlich, dass das Einst sich sowohl auf die Vergangenheit - „Einst bauten wir ein Schulhaus“ - als auch auf die Zukunft - „Einst werden wir ein Schulhaus bauen“ - richtet. Oevermann spricht von einer hierarchisch doppelten Opposition von Temporalität (Oevermann 2016: 70 f.), die an die Stelle eines linearen Modells von Vergangenheit, Gegenwart und Zukunft tritt. Denn für die Lebenspraxis zentral ist die primäre Unterscheidung von $\mathrm{Ge}$ genwärtigkeit und Nicht-Gegenwärtigkeit. Erst innerhalb dieser Differenz lässt sich in der Nicht-Gegenwärtigkeit die Opposition von Vergangenheit und $\mathrm{Zu}$ kunft unterscheiden. Die Trennung von Gegenwärtigkeit und Nicht-Gegenwärtigkeit ist trennscharf; sie hat eine Ausdehnung von null. Sie ist zudem flüchtig; sie ist nichts als Vollzug. Im Modell der Sequenzialität ist daher die Sequenzstelle S (0) durch einen Hiatus von den nicht-gegenwärtigen Sequenzstellen in der Vergangenheit und der Zukunft getrennt.

Im Gegensatz zur zeitlichen Trennung ist die räumliche Nicht-Gegenwärtigkeit im Dort nicht trennscharf abzugrenzen, denn ,das Dort ist in sich ungeschieden homogen“ (Oevermann 2003: 360). Das zeigt die folgende Darstellung (Modell nach Oevermann 2016: 72): 


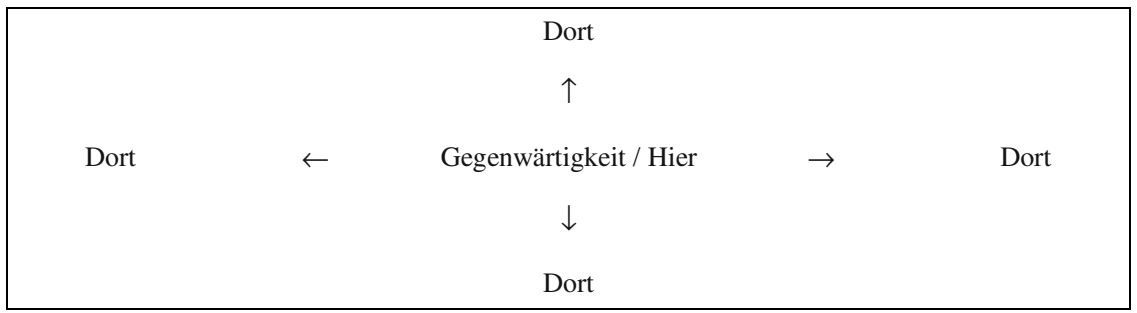

Abb. 3: Darstellung des sozialen Raums nach Oevermann (2016: 7)

Das Dort breitet sich radial um die Positionalität einer Lebenspraxis aus. Es ist nicht absolut, sondern perspektivisch abhängig von der Lage in der jeweiligen Gegenwärtigkeit einer Lebenspraxis. Da das Dort ungeschieden ist, kann keine scharfe Grenze gezogen werden.

\footnotetext{
„Darin besteht der wesentliche Unterschied zwischen Raum und Zeit, der rückwirkend auf die Nicht-Unterscheidbarkeit von Raum und Zeit für die Gegenwärtigkeit kraft der zeitlichen und räumlichen Verschiedenheit der jeweiligen Opposition von Gegenwärtigkeit und NichtGegenwärtigkeit projiziert wird. Weil die Grenze zwischen dem Hier und dem Dort nicht rückwirkend markiert ist, muss sie eigens als Grenze zwischen dem Innen (= Hier) und dem Außen (= Dort) einer Lebenspraxis gebildet werden.“ (Oevermann 2016: 74)
}

Die Positionalität der Lebenspraxis stellt sich also in einer Abgrenzung von Innen und Außen her, die in der Krise immer wieder hergestellt wird. Dies ergibt sich zwingend aus der Ungeschiedenheit des Raums in der flexiblen Ausdehnung um die Lebensmitte einer Lebenspraxis herum. Räumliche Positionalität heißt also, dass die Grenzen hergestellt werden müssen, je nach Funktion und je nach Aggregierungsniveau, die eine Lebenspraxis ausmachen. Dies geschieht in Form von In- und Exklusion, aber mitunter auch in einer physischen Positionalität, indem sich Kollektive durch Behausungen einen Sitz erstellen, der die In- und Exklusionsrechte material verkörpert.

In Plessners exzentrischer Positionalität, die insbesondere in der Architektursoziologie von Fischer (2004, 2009), aber auch bei Steets (2015) bedeutsam ist, wird eine ähnliche Konfiguration über die Körper hergestellt. Plessner sieht den Menschen doppelt in der Welt: indem er ein Körper ist und indem er einen Körper hat. Der Mensch ist von Geburt an ein Körper. Dies entspricht einer zentrischen Positionalität, die der Mensch auch mit dem Tier beziehungsweise mit jedem Objekt teilt. ,Exzentrische Positionalität‘ meint, dass der Mensch 
weder als Biowesen lebt, wie die Tiere, noch als reines Vernunftwesen, sondern ein Verhältnis zu seinem Körper entwickeln muss, welches kultureller Natur ist. Der Mensch ist nicht zentrisch im Hier und Jetzt begrenzt. Er kann eine Distanz dazu herstellen, was ihn aus seinem Körper „heraussetzt“, ohne dass er seinen Körper verlassen kann: „Sie [die Menschen, JE] stehen im Körper zum Körper im Abstand" (Fischer 2004: 13). Das bedeutet, dass die Innen-Außen-Abgrenzung als Positionalität ständig hergestellt werden muss, was einen fortdauernden Handlungsdruck auslöst. Der Mensch hat keine ruhende Position, sondern er muss sich selbst, sein Verhältnis zur Welt - zu Dingen und zu anderen Menschen - fortwährend handelnd erschaffen. Dies ist die Antriebsfeder, die zu einem nicht stillstehenden Strom an Externalisierungen führt, wie Steets es mit Rückgriff auf Berger und Luckmann ausführt (vgl. ausführlich 2015: 109 ff.). Externalisierung meint, dass der Mensch sich die Welt aneignet, indem er sukzessive subjektiven Sinn erzeugt, der sich in der Welt objektiviert. „Damit erzeugt er etwas, das ihm (...) fehlt, wenn er geboren wird, und das er handelnd erst hervorbringen muss: ein stabiles Welt- und Selbstverhältnis und eine Ordnung, die ihn in Beziehung zu anderen Menschen setzt“" (Steets 2015: 109). Denn als zentrischer Körper muss der Mensch ein exzentrisches Kulturwesen werden, damit er als Naturwesen überleben kann. Und als Kulturwesen muss er sich die Welt aneignen, indem er seine Welt schafft und seine eigene Identität in der Außendarstellung erzeugt.

Im Modell von Oevermann entsteht die Triebfeder für die Herstellung von Identität dadurch, dass die räumliche Positionalität im Gegensatz zur zeitlichen Flüchtigkeit notwendigerweise eine stabilisierende Wirkung auf die durch die Flüchtigkeit instabile Gegenwärtigkeit haben muss: Sie stellt „um des Überlebens willen (...) eine stabile, dauerhafte Verortung einer Lebensmitte [her, JE], die über ein gleichbleibendes Hier, über eine Sesshaftigkeit verfügt" (Oevermann 2003: 364). Sie ist damit Konstitutionsbedingung von Identität, die sich in der flüchtigen Gegenwärtigkeit des Jetzt auf einen stabilen und dauerhaften Innenraum der Sesshaftigkeit im Hier bezieht. Dabei bedeutet Sesshaftigkeit nicht eine physisch stabile Lokalisierung, sondern eine „Verankerung in einer vergemeinschafteten Form von Sozialität“ (Oevermann 2003: 363). 


\subsubsection{Die räumliche Konstitution von Positionalität, Subjektivität und Identität}

Der Zusammenhang von Subjektivität und Identität zeigt sich in der strukturtheoretischen Konzeption in der Dialektik zwischen Krise und Routine. Subjektivität ist unmittelbar an die Gegenwärtigkeit des Augenblicks geknüpft und realisiert sich als innere Erfahrung in der Krise. Sie bildet die Innenseite der Positionalität und ist von außen nicht zugänglich. Identität hat demgegenüber einen routinehaften Charakter und stellt eine stabile, kohärente und konsistente Basis der Selbsterfahrung dar. Identität ist nicht starr, sondern kann sich jederzeit transformieren. Räumlich betrachtet ist die Identität die Außenseite der InnenAußen-Abgrenzung. Deshalb muss sich eine Lebenspraxis auch zwangsläufig nach außen selbst inszenieren beziehungsweise repräsentieren. Sie tut dies in der Selbstdarstellung über kulturelle Artefakte, die die Identität der Lebenspraxis symbolisch nach außen abbilden. Identität muss krisenhaft und grenzrealisierend hergestellt werden, da die „Nacktheit“, die die exzentrische Positionalisierung mit sich bringt, nur über „die Grenzrealisierung des ,Kleides“ verringert werden kann" (Fischer 2004: 14, Hervorhebung im Original). Dabei gilt dieser Darstellungszwang auch für kollektive Entitäten, für welche das Kleid z. B. einen Sitz, also ein Gebäude darstellt.

In der Kurzform kann konstatiert werden, dass sich Subjektivität zeitlich gesehen zwischen alter Identität und neuer Identität in der Gegenwärtigkeit realisiert (vgl. ausführlich Wagner 2004: 148 ff.). Eine stabile kohärente Subjektivität im Innern, welche eine stabile kohärente Identität nach außen externalisiert, verlangt zwangsläufig nach einer stabilisierenden Wirkung der Positionalität einer Lebenspraxis über die Sesshaftigkeit.

Dieser Umstand spiegelt sich auch in Meads (1998) Identitätskonzeption von I, me und self. Das self als Identität des Einzelnen ergibt sich aus der Spannung, in der der Mensch selbstreflexiv die Subjektivität des $I$ in einem Perspektivwechsel aus der Sicht des generalisierenden Anderen - des me - betrachtet. Die Perspektivenübernahme, in dem sich ein Subjekt von außen betrachtet, kann nur räumlich vorgestellt werden in einer Betrachtung, in dem sich das $I$, das sich im gegenwärtigen Hier befindet, gedankenexperimentell in die Perspektive des Dort versetzt. Als stabiles self verkörpert dieses die Positionalität beziehungsweise Sesshaftigkeit einer Lebenspraxis. Dies ist insofern nicht nur für die Kon- 
zeption der flüchtigen Subjektivität bedeutsam, sondern auch für die Beständigkeit der eigenen Identität. Das menschliche Handeln ist wesentlich dadurch bestimmt, dass der Mensch in einer antizipierten Perspektivenübernahme sein eigenes Verhalten wie ein äußerliches Objekt betrachtet. Oder anders gesagt: Erst in der Interaktion mit anderen Subjekten verfügt der Mensch über die Möglichkeit der Selbstreflexion. Und diese ist Voraussetzung dafür, den subjektiven Sinn in einer Form zu externalisieren, dass nach außen eine konsistente und kohärente Identität dargestellt und objektiviert wird, zum Beispiel in Form eines Sprechakts oder eines Gebäudes.

Die Identitätsentwicklung in der intersubjektiven Interaktion ist nicht nur Bedingung für die Selbstreflexion, sondern auch dafür, dass wir einen reflexiven Umgang mit Dingen entwickeln (Steets 2015: 232 bzw. 242). Dabei hat der Aneignungsprozess auch die umgekehrte Richtung, wie Steets ausführt:

„Damit ein Ding wie beispielsweise ein Haus zu meinem Haus wird, ich es also internalisiere, muss ich mich selbst in ihm wiederfinden. Das gelingt am besten, wenn ich das Haus nach meinen Relevanzen verändere oder ergänze, wenn ich also subjektiven Sinn externalisiere." (2015: 244)

Positionalität verkörpert und stabilisiert die Praxismitte einer Lebenspraxis. Je nach Handlungssubjekt, also je nach Aggregierungsniveau der Lebenspraxis, ist der Radius, der das Innen und Außen konstituiert, unterschiedlich weit gefasst. Man kann sich ein idealtypisches Schalenmodell in einem dreidimensionalen Raum vorstellen, in dessen Mitte die Lebenspraxis eines Einzelsubjektes sitzt, um die herum sich die verschiedenen Innen-Außen-Abgrenzungen denken lassen (siehe dazu Schmidtke 2006: 49 f.). Dabei ist bezeichnend, dass in den nicht leiblich-körperlich gebundenen Schichten, so zum Beispiel in der Architektur, eine Sesshaftigkeit geschaffen wird, die auch dann wirkmächtig ist, wenn sich das betreffende Subjekt außer Haus befindet. Doch soll der Fokus vorerst auf die radial angeordneten Schichten der Innen-Außen-Abgrenzungen gerichtet werden.

Als erste und wichtigste Abgrenzung ist bei Individuen die Haut als Grenze und Konstituierung der leiblichen Positionalität zu nennen. Sie teilt trennscharf das Außen vom Innen eines Individuums. Die räumlich-leibliche Abgrenzung durch die Haut wird mit den Tieren geteilt. Der Unterschied ist in der inneren Positionalität zu sehen, die sich erst durch Sozialität und Sprache herstellt und in 
welcher die Sesshaftigkeit verankert ist, welche der „Keimling von Subjektivität“" (Oevermann 2016: 94) ist. Zugleich stellt sich in der Positionalität auch die Identität nach außen her. Daher ist die Haut ein wichtiges Medium, auf der sich eine Identität expressiv abbilden lässt. Oder umgekehrt ist die Beschaffenheit der Haut, z. B. die Farbe, für die Identitätszuschreibungen zentral.

Die an die Haut anschließende und erweiterte Innen-Außen-Abgrenzung einer individuellen Lebenspraxis stellen die Kleider dar, die je nach Schichten auch unterschiedliche Nähebereiche der Intimität abgrenzen. Das Kleid bietet einerseits einen Schutz. Es bietet aber vor allem die Möglichkeit, sich eine Außendarstellung zu geben. Turner bezeichnet deshalb die Kleider als „soziale Haut" (Turner 2012). Das Kleid ist damit auch Ausdruck einer eigenen Welterrichtung. Kleider resultieren aus Kulturtechniken. Sie sind kultur- und ressourcenabhängig und sind eng mit den technologischen Möglichkeiten einer Gesellschaft verbunden. Als Kulturtechniken sind Kleider zugleich symbolisches Trägersystem einer Repräsentationsfunktion: ,Kleider machen Leute“ sagt das Sprichwort. Kleider markieren die Funktion und den Status einer Lebenspraxis und verweisen damit auf ein Set von Rechten und Pflichten, die Trägerin und Träger innehaben. Man denke zum Beispiel an die Schuluniformen: Diese symbolisieren die Uniformität der Adressierung der Schüler_innen als Gleiche.

Die nächstäußere Abgrenzung der leiblichen Einzelsubjekte bilden die Behausungen. Häuser verkörpern den Sitz von Clans, Familien oder anderen kollektiven Gemeinschaften sowie Organisationen. Sie sind die verkörperte Sesshaftigkeit von vergemeinschafteter Lebenspraxis, in der das einzelne Subjekt eingebettet ist. Oder umgekehrt ausgedrückt: Architektur ist die Antwort auf die Krise der Sesshaftigkeit einer Gemeinschaft. In diese eingelagert ist die Krise der Sesshaftigkeit des Einzelsubjektes. Letzteres ist der Ersteren immer inhärent. Als Sitz einer gemeinschaftlichen Positionalität sind Behausungen für die Stabilität der Identität zentral, da sie dauerhaft und objektiviert sind. Nicht von ungefähr wird beispielsweise im Kontext der Bildungsforschung oft vom Elternhaus gesprochen, wenn die sozialisatorische Funktion der Herkunftsfamilie gemeint ist (z. B. Egger et al. 2016; Hummrich \& Wiezorek 2005). Von den Behausungen und der Architektur wird jedoch noch ausgiebig zu sprechen sein.

Als nächste Innen-Außen-Abgrenzung um die Behausung herum sind die Siedlungsgebiete zu nennen, zu denen sich ein Leib, aber auch ein Haus oder sonstiger architektonischer Körper in Beziehung setzen muss: der Weiler, der 
Straßenzug, das Dorf, das Quartier. In der raumtheoretischen und sozialgeographischen Diskussion wird meist vom Sozialraum oder der Sozialräumlichkeit gesprochen, wenn die Betrachtung eines größeren Siedlungszusammenhangs als sozioökonomisches und/oder kulturell-historisches Gepräge im Blick ist (z. B. Kessl \& Reutlinger 2008, 2010). Diese sind ihrerseits wiederum innerhalb von größer aggregierten Gebilden wie der Stadt, der Region, der Nation bis hin zu supranationalen Gebilden verortet. Sie alle müssen jeweils eine Innen-AußenAbgrenzung herstellen, um eine im Innenraum verankerte Positionalität zu erzeugen, die Kern einer handelnden Subjektivität ist und zugleich als Außenabgrenzung eine stabile Identität ausbildet. Die Abgrenzungen abstrakter Gebilde wie eine Stadt oder auch eine Nation oder supranationale Organisationen sind auf eine durch Architektur hergestellte Innen-Außen-Abgrenzung angewiesen in Form sichtbarer Grenzziehungen über Marksteine, Schilder usw.

Jede Gemeinschaft muss sich einen räumlich abgesteckten Rahmen geben, der sich in der Außendarstellung als spezifische Form der Selbstinszenierung in einem ästhetischen Ausdruck ausformt (ausführlicher siehe Loer 2013: 13). Der Rahmen fungiert sowohl als Innen-Außen-Abgrenzung: In Form von materialisiert-persistenten Manifestationen sind Quartiere, Städte und Nationen entweder entlang natürlicher Grenzen oder durch Grenzsteine und -zäune, Einfriedungen, Stadtmauern usw. markiert. Zum anderen repräsentiert sich eine kollektive Identität auch über die Emergenz einer symbolischen spezifischen Architektur, die eine bestimmte gemeinschaftliche Lebenspraxis repräsentiert und damit für die Gemeinschaftsidentität wesentlich ist. So repräsentieren Architekturen auch Gesellschafts-, Clan-, Stadt- oder regional-spezifische Architekturen und symbolisieren stadt- und landestypische Elemente wie beispielsweise das Emmentaler Bauernhaus, die Lauben der Zähringerstädte u. v. m. Dazu gehören aber auch die spezifischen Gebäudetypen wie Schulhäuser. Dies zeigt sich explizit auch in der normativen Diskussion, die die Entstehung des Gebäudetyps Schule dominierte (Helfenberger 2013). Ein zentrales Anliegen der Musterpläne war, dass der Entwurf ein Gebäudetyp sein sollte, der in seiner Funktionalität einheitlich ist, sich in seiner äußerlichen Gestalt aber den jeweils regionalspezifischen Architekturen angleichen kann. Trotzdem hat die Schulhausarchitektur interessanterweise die Herausbildung einer landesweiten Formsprache angestossen und beschleunigt. Die Normierung von Gebäuden, die mit den in der Schweiz als ,Normalien` benannten Musterplänen einhergeht, hat gemäß Knutti-Baumann 
(1987) dazu geführt, dass sich eine landesweite ,nationale“ Architektursprache entwickeln und verbreiten konnte.

Das radiale Schalenmodell von Innen-Außen-Abgrenzungen ist nicht als statisch zu verstehen. Die Innen-Außen-Abgrenzungen sind tendenziell umso stabiler, je größer die Entität ist: Landesgrenzen haben länger Bestand als Stadtgrenzen, die wiederum weitaus dauerhafter sind als die Parzellen einzelner Häuser usw. Im Prinzip können aber alle Sesshaftigkeiten in ihren Innen-AußenAbgrenzungen - die in der Theoriesprache der Objektiven Hermeneutik als Routinen gefasst werden - jederzeit in eine Krise geraten und sich transformieren.

\subsubsection{Architektur als objektivierte Materialität des Sozialen}

Die Innen-Außen-Abgrenzung durch Architektur hat weitreichende Auswirkungen auf die anthropologischen und phylogenetischen Entwicklungen humaner Praxis, die hier aber nur so weit als absolut notwendig thematisiert werden sollen (vgl. dazu an Stelle vieler Klotz 1995; Rykwert 2005; Schmidtke 2006). Die phylogenetischen Entwicklungen sind dort relevant, wo sie zur Bestimmung der konstitutiven Elemente von Architektur als Sozialem und damit für die Fragen von Architektur als Protokoll und Forschungsgegenstand weiterführend sind.

Entscheidend ist, dass die flüchtige und jeweils immer wieder herzustellende Innen-Außen-Abgrenzung durch Architektur dauerhaft gemacht wird. Damit wird eine zusätzliche Innen-Außen-Abgrenzung, quasi eine ,dritte Haut‘ erbaut.

„Zur leiblichen Innen-Außen-Abgrenzung und zur Innen-Außen-Abgrenzung, die sich in einer Praxisraumzeitlichkeit vollzugsgebunden realisiert, wird eine zusätzliche Innen-AußenAbgrenzung gewonnen, die vorher allenfalls aufgrund landschaftsmorphologischer Gegebenheiten (Höhlen, Täler, natürliche Barrieren) zum Tragen kam.“ (Schmidtke 2006: 58 f.)

Dabei sind zwei Effekte wesentlich. Durch Architektur bekommt die subjektiv flüchtige Lebenspraxis des Vollzugs eine objektivierte Ausdrucksmaterialität. Der zweite Effekt ist, dass diese Objektivationen vergleichsweise dauerhaft sind. Sie sind in der Perspektive der endlichen Lebenspraxis der Subjekte für die Ewigkeit erstellt. 
Dies bringt gewisse Analogien zur Schrift mit sich. Wie die Schriftlichkeit eine Externalisierung von Subjektivem darstellt und das Schriftstück eine Objektivation dieser Subjektivität darstellt, stellt Architektur eine Externalisierung und Objektivation des Sozialen dar. Dieser Umstand kann zur Klärung des Verhältnisses von physischem Raum und sozialem Raum herangezogen werden. Architektur verhält sich zum Raum ähnlich wie die Schrift zum Papier. ${ }^{5}$ Der Raum an sich ist aus einer soziologischen Perspektive bedeutungsleer. Seine Funktion liegt in der Trägerstruktur und dem ihm inhärenten Potenzial, das er in Form von topographischen Merkmalen wie etwa Höhlen oder natürlichen Grenzen birgt, die von den Menschen als Ressourcen angeeignet werden können. $\mathrm{Zu}$ einem sozialen und damit sinnstrukturierten Raum wird er erst in der dauerhaften Aneignung, die mit einer symbolisch vermittelten sinnhaften Markierung einhergeht. Auch die anthropologische Morphologie weist auf den Umstand hin, dass Bedeutung dem Raum erst durch Handlungen im und mit dem Raum zukommen. Raum hat nicht per se eine Bedeutungsfunktion, indem sich Siedlungen dort entwickeln, wo der Raum gewisse Strukturen oder Ressourcen bereithält. Die räumlich gebundenen Ressourcen sind in Abhängigkeit der technologischen und sozioökonomischen Möglichkeiten einer Gesellschaft zu sehen und werden erst bedeutsam, wenn die soziale Organisation einer Gesellschaft sie sinnhaft einbindet (Mauss 2004; Mauss \& Lévi-Strauss 1974).

Raum wird als sozialer Raum durch Handlungen hervorgebracht. Und dabei ist Architektur das wesentliche Mittel, mit dem der Raum ,beschrieben “ wird beziehungsweise der dem Raum Sinn- und Bedeutungsstrukturen eingraviert. Analog zum Papier, das erst mit der Beschreibung sinnhafte, das heißt ausschließlich lesbare Bedeutungsstrukturen erhält, erfährt auch der leere Raum erst mit der Bebauung eine Realitätserweiterung, die über die topographischen Elemente des Raums hinausgehen. Der soziale Raum enthält mit Architekturen soziale Elemente, die erfahren und gelesen werden. Durch humane Praxis, also durch die Bearbeitung und Gestaltung mittels sozial erstellter Bauwerke und

5 Dabei gibt es zentrale Unterscheidungen zur Schrift. Insbesondere ist Architektur omnipräsent. Als Kommunikationsmedium ist sie ein ,schweres Medium“ (Fischer 2017: 57). Sie ist von Individuen unveränderlich und unhintergehbar. Der bebaute Raum ist die wesentliche Erfahrung der Welt. Man kann sich ihr nicht entziehen. 
Bodenbewirtschaftungen ${ }^{6}$ schreiben sich die Sinn- und Bedeutungsstrukturen dem Raum ein. Anders gesagt: In gemeinschaftlichen kulturellen Praktiken der Erbauung von Architekturen realisiert die humane Spezies Objektivationen von symbolisch vermittelten Repräsentationen seines Selbst. Deshalb ist für LéviStrauss (1981: 293 ff.) die Entdeckung der Schriftlichkeit nicht die Erklärung für die schöpferischste Phase in der Geschichte der Menschheit. Die Schrift entwickelt sich erst Jahrtausende später, in denen mit Architekturen, Landbau und Domestizierung von Tieren experimentiert wurde. Auf technischer Ebene sind es die Fähigkeiten zur Erbauung von Architekturen, die die Voraussetzung für die Emergenz und Ausdifferenzierung der vielfältigen humanen Praxisformen im Neolithikum darstellen. ${ }^{7}$

Umgekehrt muss die Architektur gelesen werden, wenn die Sinnhaftigkeit in ihren Objektivationen verstanden werden soll. Man muss, wie Steets es ausdrückt,

„die Sprache dieser Welt lernen, Relevanzen nachvollziehen, Institutionen verinnerlichen und so weiter. Auf die gebaute Umwelt bezogen heißt das nichts anderes, als dass ich lernen muss, räumliche Situationen, Gebäudeformen, Fassaden, Proportionen, Abstände, ornamentale Verzierungen etc. zu lesen." (Steets 2015: 185, Hervorhebung im Original)

Was die humanen von tierischen Architekturen unterscheidet, ist, dass sie eine symbolische Repräsentation der ,bewohnenden“ Lebenspraxis ist. Nest- und Höhlenbau schützen Tiere rein funktional vor Wetter, Klima, Feinden usw. Demgegenüber ist Architektur als Antwort auf die Krise der Sesshaftigkeit zu sehen, in der sich eine kollektive Lebenspraxis zwangsläufig selbst inszenieren muss.

Denn die Objektivation von Bedeutungsstrukturen in Form von Architektur ist für die Aufrechterhaltung einer Gemeinschaft mehrfach essenziell. Gesellschafts- und Architekturformen sind so miteinander verwoben, dass Ersteres in Letzterem aufgehoben ist. So zeigt Lévi-Strauss' Analyse der Bororo, wie sich sowohl die Mythen als auch die Soziogrammatik der Verwandtschaftsstrukturen in der Anordnung der Architekturen ,,in einer grandiosen Kosmologie“ nieder-

$6 \quad$ Sprachlich drückt sich dies aus, indem durch Bewirtschaftung Land zu Kulturland wird.

7 Die potenziellen Baumaterialien sind wesentlich vielfältiger als die potenziellen Schreibutensilien, die ihrerseits bereits eine kulturell hochentwickelte Gesellschaft voraussetzen. 
schlagen, die von den Weisen ,im Plan ihrer Dörfer und in der Verteilung der Wohnstätten niedergelegt“ (Lévi-Strauss 1981: 236) worden waren. ${ }^{8}$ Erst als Objektivationen schaffen Architekturen eine objektive Realität und damit ein „kollektives Gedächtnis“ (Halbwachs 1997); also Geschichte, was wiederum als Analogon zur Schriftlichkeit gesehen werden kann. Eine Gemeinschaft ohne Architektur und damit ohne material-persistente, dauerhafte Sesshaftigkeit muss die Vergemeinschaftung und ihre Geschichte immer wieder im Vollzug herstellen. ${ }^{9}$ Eine größere stabile Gemeinschaft ist dabei kaum vorstellbar.

Architektur wirft auch die Frage der eigenen Herkunft auf. Sie erzwingt eine Auseinandersetzung mit dem eigenen Erbe, wenn es darum geht, dem bestehenden Gefüge ein neues Gebäude hinzuzufügen. Mit der objektivierten Realität der Geschichte in Form von Überresten alter Siedlungen, aber auch von gegebenen und zukünftigen Bauten, stellt sich zwangsläufig das Problem der Bewährung (ausführlich Oevermann 2001, 2009a). Die Auseinandersetzung mit der Herkunft ist dabei zwingend auch eine Auseinandersetzung mit der Zukunft. Denn es wird zugleich die Frage aufgeworfen, was den zukünftigen Generationen überlassen werden soll, und zwar sowohl in Bezug auf die Spurenreste vorhandener Geschichte als auch in Bezug auf die Gestaltung der produzierten Architektur, also des eigenen Praxisvollzugs. Letztere untersteht damit einem nicht-stillstellbaren Bewährungsdruck (vgl. Oevermann 2001).

Schulbauten haben in dieser Hinsicht für die neuere Geschichte eine herausragende Bedeutung. Dies ist zum einen dem Umstand geschuldet, dass Schulbauten den gesellschaftlich zentralen Bereich von Bildung beheimaten. Damit geht aber auch einher, dass Schulhäuser als staatliche Repräsentationen und wichtige staatliche Bauaufgabe dem Nationalstaat ein Gesicht geben. Es sind nicht nur historische Monumente. In den jeweils gegenwärtigen Entstehungskontexten sind es wichtige identitätsstiftende Bauten der jeweiligen Ge-

8 Das Wissen über die Macht von Architekturen haben sich auch die Missionare zunutze gemacht, indem sie den Stämmen Häuser gebaut haben. Nachdem die Eingeborenen in die für sie gebauten Häuser gezogen sind, verloren sie ihre soziale Orientierung. Die soziale Ordnung und Gemeinschaft brach in der Folge in sich zusammen.

Die Innen-Außen-Abgrenzung wird bei lose verbundenen Gruppen durch Vollzug hergestellt. Bei Empfängen, Kongressen usw. bilden sich kleine Gruppen, denen man sich nur nach gewissen Regeln nähern beziehungsweise an denen man teilnehmen darf, ohne dass die Sittlichkeit verletzt wird (Schmidtke 2006: 50). 
meinschaften, die der Legitimität von Herrschaft dienen. ${ }^{10}$ Nicht zuletzt sind Schulbauten als Bauten für Kinder Orte der Sozialisation, deren Sinnstrukturen wir internalisiert haben. Haben wir doch selbst alle in den Schulhäusern Tausende von Stunden unseres Lebens verbracht und deren Sinngehalte internalisiert. Sie erhalten eine Bedeutsamkeit, die als Erinnerungsspuren internalisiert sind, die beim Betreten von Schulbauten wiederum abgerufen werden.

Dies verweist auf eine weitere Bedeutungskonstellation: Zentral für die Herausbildung einer stabilen Gesellschaftsordnung ist aus einer makrosoziologischen Perspektive die Architektur als Speicher von implizitem Wissen, von Ordnungsstrukturen und sozialen Regeln.

Aus einer mikrosoziologischen Perspektive geschieht dies, indem Architektur als Speicher von Regeln eine handlungsleitende Bedeutungsstruktur innehat und für das Kollektiv und die Individuen eine stabilisierende Wirkung auf die Handlungen und die Identität generiert. Erst über Architektur wird es möglich, dass die Verbundenheit eines Subjektes mit dem Innenraum einer Sesshaftigkeit, z. B. einer Schule oder einer Nation, auch dann als Einheit verbunden bleibt, wenn es sich außerhalb dieser Mitte, also außerhalb des Hauses oder des Landes befindet.

Die Sesshaftigkeit ist äußerst stabilisierend und für die Emergenz von aggregierten Lebenspraxen zentral. Dies erscheint zunächst trivial und zirkulär, da Häuser als Sesshaftigkeit von gemeinschaftlichen Lebenspraxen definiert wurden. Es ist aber insofern nicht trivial, als es Bedingung für die Herausbildung kollektiver Identitäten, für die Ausdifferenzierung von komplexeren Gemeinschaften, der Ausbildung von Institutionen und der Konstitution von Gemeinschaft und Gesellschaft ist. Vorderhin aber, um bei der einzelsubjektiven Perspektive zu bleiben, hat dies zur Folge, dass das Hier auch im Dort verankert wird. Die leibliche Positionalität, die krisenhaft im Hier hergestellt wird, stabilisiert sich, indem sie in einem Dort in einer stabilen dauerhaften Routine verankert ist.

10 Deshalb ist es für das subsidiäre politische System bedeutsam, dass die Schulen in der Schweiz zwar von den Kantonen betrieben werden, die bauliche Infrastruktur aber in der Verantwortung der kleinsten lokalen politischen Gemeinschaften, den Gemeinden, liegt. 
Die gebaute Sesshaftigkeit hat weitreichende Folgen. Beispielsweise müssen Eingänge so gestaltet werden, dass für den Außenstehenden sichtbar verkörpert wird, wie eine Interaktion und eine gemeinsame Praxis vollzogen wird (Schmidtke 2006: 51). Die Hüllen sind als Eröffnungsprozeduren zu lesen. Fassaden und ihre Erschließungen sind nicht nur als Eröffnungsprozeduren von Interaktionen und gemeinsamer Praxis, also als Vermittlung von innen und außen gegenüber potenziell Fremden zu lesen. Behausungen emergieren gemeinschaftliche Regeln und Normen der Nutzung und der Berechtigungen.

Architektur geht immer mit Inklusions- und Exklusionsregeln einher, die spezifische Nutzungsrechte für unterschiedliche Gruppen architektonisch markieren. Am Beispiel von Schulhäusern markiert Architektur über die Einfriedungen und die Positionalität von Gebäuden, Zugängen usw. die Nutzungsrechte für spezifische Gruppen. Schulanlagen sind - obwohl es sich per se um einen öffentlichen Raum handelt - implizit nur für Schüler_innen und Lehrpersonen zugänglich. Auch Eltern sind, zumindest in der schweizerischen Volksschule, ${ }^{11}$ auf dem Schulareal unerwünscht, was für weitere erwachsene Personen noch verschärft gilt. Diese ,Betretungsverbote“ sind - heute - jedoch ungeschriebenes Gesetz und als implizite Regeln internalisiert. Zudem variieren sie zeitgebunden, je nachdem ob Unterricht stattfindet oder nicht. Dabei sind die Inklusions- und Exklusionsregeln Leistungen des Praxisvollzugs. Die Architektur stellt lediglich den Rahmen oder die Grenze dar, an der sich die Vollzugsleistung realisiert. Mit der Feststellung von In- und Exklusion und der Frage der Vermittlung zwischen innen und außen wird die Konstitution eines Innen und Außen bereits vorausgesetzt. Mit der Abgrenzung schafft Architektur zwangsläufig eine Unterscheidung von privatem Leben und öffentlichem Leben. ${ }^{12}$ Die Schaffung von Privatheit und Öffentlichkeit ist ein konstitutives Merkmal von Architektur, das sich in allen Raumtheorien der Moderne wiederfindet. Wie bereits erwähnt wurde,

11 Wobei es auch hier Unterschiede zwischen deutschsprachigen und frankophonen Landesteilen gibt: In der Romandie gilt das implizite ,Betretungsverbot‘ ungleich schärfer als in der Deutschschweiz, wo Eltern zumindest während der ersten Wochen auf dem Schulareal noch geduldet werden.

12 Schmidtke (2006) legt überzeugend dar, dass sich diese Unterscheidung zwangsläufig und universell konstituiert. Die Unterschiede sind lediglich darin zu sehen, dass Privatheit und Öffentlichkeit vor der Neuzeit eine(n) andere(n) Stellenwert und Bedeutung hatten und dass die Grenzen anders gezogen wurden (vgl. auch Lang 2010). 
bekommt Architektur damit zwei Analyserichtungen: Nach innen befinden sich die Lebensmitte, die Sesshaftigkeit, das Private, die Binnenbeziehungen. Zudem ist der Innenraum gegliedert bezüglich der Funktionalität, für die ein Gebäude erstellt ist. Nach außen hat die Hülle die Funktion, die Lebenspraxis und die Bauherrschaft des Gebäudes zu repräsentieren.

\subsubsection{Der Auftrag eines Gebäudes - Binnenfunktion von Architektur}

Der Innenraum eines Gebäudes hat eine größere Affinität zur Funktion des Gebäudes als die Außengestaltung. Die Binnengliederung ist explizit für den Auftrag des Gebäudes geplant und gebaut. Sie ist funktional, wobei die „Funktionalität (...) die methodisch kontrollierte Thematisierung eines bestimmten Verhältnisses von Flüchtigkeit, von Praxisvollzügen zu ihrer Entsprechung in einer nicht-flüchtigen starren Raumgliederung" (Schmidtke 2006: 56) ist. Anders gesagt: Architektur besteht aus einem ,Raumprogramm“ und einem ,Programm der Räume', die besonders dauerhafte Routinen mit dem Zweck der Herstellung von Dispositionen sind. Architektur fördert spezifische Praktiken, z. B. das Unterrichten in seiner historisch-gesellschaftlich gedachten Form, und sie soll gewisse Handlungen explizit oder implizit auch verhindern. Indem ein Gebäude den Möglichkeitsraum der Praktiken - als eine von vielen Einflussgrößen im Erzeugungsparameter - strukturiert, kann sie die Autonomie einer Praxis befördern oder tendenziell verhindern. Sie kann eine möglichkeitseröffnende Wirkung haben und damit das Innovationspotenzial befördern, oder sie kann tendenziell eine Zweckprogrammierung darstellen und damit den autonomen Möglichkeitsraum einschränken. In dieser Spannweite ist Architektur bezüglich ihrer Funktionalität zu betrachten.

Die Binnengliederung von Gebäuden - also das Raumprogramm, die Raumanordnungen und das Programm der Räume - kann als explizite ,Theorie“ über den Auftrag des Gebäudes gelesen werden. Das Raumprogramm eines Schulhauses stellt eine realisierte ,Theorie der Schule' dar, die über Funktionsanforderungen und die Programmatik von Schule Auskunft gibt. Aber auch die Raumanordnungen als Binnengliederung der ,Privatheit" der Lebenspraxis können als Spiegel beziehungsweise als Innenlogik der Beziehungsstrukturen gelesen werden: Für welche Akteure wurde ein Schulhaus gebaut, und wie werden 
die Binnenbeziehungen dieser Akteure gedacht, geplant, konfiguriert und disponiert?

Wesentlich ist, dass die Binnengliederung nicht nur material-persistent zu lesen ist. Räumliche Ordnungen sind mit sozialen Bedeutungen gekoppelt. Die Innen-Außen-Abgrenzung schafft einen Innenraum, der auch im einfachsten Falle einer leeren Hülle eine Innengliederung durch die Differenzierung über die phänomenologischen Kriterien von oben/unten, hinten/vorne und links/rechts erfährt. Diese Unterteilung ist mit sozialen - oft dichotomen - Gegensätzen gekoppelt, die nicht allein technischen Anforderungen oder funktionellen Notwendigkeiten geschuldet sind, sondern mit sozialen Merkmalen korrespondieren wie männlich/weiblich, erwachsen/kindlich, hohem/tiefem sozialem Status usw. Die Innengliederung eines Hauses ist notwendigerweise auf das Außen bezogen. Laut Bourdieu spiegeln sich im Innern die Beziehungen, welche die bewohnende Lebenspraxis zum Außen hat (vgl. dazu die Analyse des kabylischen Hauses bei Bourdieu 1976). Aus den bisherigen konstitutionstheoretischen Erörterungen kann abgeleitet werden, dass der Innenraum und die Strukturierung des Innenraums immer in Bezug auf die Verhältnisse zum Außen stehen.

\subsubsection{Repräsentation durch Architektur - Die Außenfunktion}

Als Fassade nach außen kommt der Architektur die Funktion der Repräsentation und Selbstinszenierung der Bauherrin beziehungsweise der Bewohner_innen des Gebäudes zu. Repräsentation ist dabei nicht eine Eigenschaft, die Architektur hat. ${ }^{13}$ Und noch weniger ist sie rein machttheoretisch als Darstellung eines Rangs und/oder von Macht zu sehen. Die Realisierung von Architektur als monumentale Geste und erdrückende Machtpräsenz ist lediglich als eine der Spielarten und Objektivierung der Selbstinszenierung einer spezifischen Gemein-

13 In der Architekturtheorie wird die Repräsentationsfunktion oft als überlieferter Begriff verstanden, da er mit Monumentalbauten verbunden ist (Gleiter 2015: 47). Ähnlich argumentiert Kemp, wenn er mit Le Corbusier von der Abschaffung der Fassade als Vermittlungsinstanz argumentiert und diese nur noch funktionalistisch sieht. Soziologisch gesehen ist Repräsentation für Architektur konstitutiv, auch dann, wenn sie im subjektiven Sinn der Architekten negiert wird. Sie repräsentiert damit diese Negation. 
schaft und natürlich als Mittel der Machtdurchsetzung und -zementierung sowie der Herrschaftslegitimierung zu sehen.

Soziologisch betrachtet gibt die Fassade und deren ästhetische Gestaltung der Funktionalität eines Gebäudes und der wohnhaften Lebenspraxis ein Gesicht. Doch ist die Repräsentation nicht auf die Symbolisierung der Funktionalität zu reduzieren, sondern sie ist zugleich Vollzug gemäß der immanenten Strukturgesetzlichkeit des Repräsentierten. So findet zum Beispiel „die Strukturierung jeglichen Handelns durch Eröffnung und Beschließung (...) in der räumlichen Unterscheidung von vorne und hinten eine Entsprechung" (Schmidtke 2008: 7). Die der Öffentlichkeit zugewandte Fassade ist als sinnhafte Eröffnungsprozedur $\mathrm{zu}$ interpretieren. Als eine sich reproduzierende Typik von Schulbauten zeigt sich, dass die Erschließungen oft auf der abgewandten Seite liegen, was einen privaten Außenraum konstituiert. Die Erschließungen sind aber oft fast verdeckt, und damit konstituieren die Schulbauten eine Bedeutungsstruktur, die sie als nicht öffentlich zugängliche Gebäude auszeichnen, obwohl sie in ihrer Formsprache öffentliche Gebäude sind.

Zentral scheint mir, dass die Hülle nicht lediglich als Ausdrucksrepertoire analysiert wird, mit dem sich eine Lebenspraxis gegenüber der Öffentlichkeit darstellt. Sie trägt sinnhafte Bedeutung. Auch das Credo der modernen Architektur, form follows function, ändert an dieser Tatsache nichts, sondern ist viel eher Ausdruck davon. Eine Architektur, die sich programmatisch der ästhetischen Gestaltung verweigert, verkörpert darin allenfalls das Misslingen der Repräsentation. Architektur realisiert aber im Gelingen oder Misslingen die ästhetische Verfasstheit der Lebenspraxis und der Funktionalität eines Gebäudes. Für die vorliegende Arbeit ist festzuhalten, dass die Unterscheidung zwischen Zweckbau und Repräsentativbau, wie sie oft auch bei der Diskussion um Schulhäuser geführt wird (z. B. Schneeberger 2005), für die soziologische Betrachtung obsolet ist. Der Unterschied ist vielmehr interpretationsbedürftig und als Wandel von Gesellschaft- und Strukturtransformation der Institution Schule zu deuten.

Mit zur Außenfunktion der Architektur gehört, dass sich Architektur zwangsläufig zu ihrer Umgebung positioniert. Die Positionierung nach außen ist gleichzeitig eine Binnengliederung der nächsthöheren Entität. Die einzelnen Gebäude konstituieren eine Schulanlage, die innerhalb eines Dorfes oder eines Straßenzuges verortet sind, die wiederum ein Quartier strukturieren, das seinerseits als Binnengliederung eines Stadtteils, einer Stadt, einer Region usw. be- 
trachtet werden kann. In der sozialräumlichen Betrachtung einer Positionalität sind die einzelnen Häuser als das innere Gewebe einer Struktur zu sehen, die als Einflussstruktur kollektive Praktiken mitprägt, produziert und reproduziert.

\subsubsection{Wirkungsweisen von Architektur}

Einer strukturalistischen Analyse ist eigen, dass zuerst die Frage nach dem ,Was', und erst dann nach dem ,Wie' gestellt wird. Daher waren bis anhin die Fragen leitend, welche das strukturelle Problem von Architektur behandelten. Architektur wurde in dieser Konzeption als je einzelfallspezifisch zu realisierende Lösung des strukturellen Handlungsproblems der Sesshaftigkeit einer kollektiven Lebenspraxis rekonstruiert. Sie ist die Ausdrucksmaterialität, in der sich eine symbolisch vermittelte Selbstinszenierung und Selbstrepräsentation einer Gemeinschaft in Bezug zu ihrer Funktionalität realisiert. Durch Materialisierung als kollektives Gedächtnis werden symbolisch vermittelt handlungsleitende Routinen institutionalisiert und die soziale Identität und Ordnung damit stabilisiert.

Im Folgenden wird diskutiert, wie Architektur ihre Wirkungen entfaltet. Dies ist aus einer allgemein architektursoziologischen Perspektive klärungsbedürftig. Es ist auch für die Analyse von Schulbauten zentral, da dies Bauten sind, in denen Sozialisation und Bildung stattfindet. Deren Auftrag besteht explizit darin, dass die Schüler_innen sich darin die gesellschaftlichen Werte und Normen, das gesellschaftliche Wissen u. a. m. aneignen. Es geht um die Frage, wie und welche Formen der sekundären Sozialisation über die gebaute Umgebung internalisiert werden.

Die Wirkungsweisen von Architektur sind erstaunlich wenig erforscht. Sie sind auch komplex und schwer zu fassen. Sie können hier nur ansatzweise skizziert werden. Es verbleibt notwendigerweise ein abstrakter Entwurf, der konstitutionstheoretisch und methodologisch wenig ausgereift ist und u. U. mehr Fragen aufwirft als beantwortet. Letztlich wird sich das Vorgehen in den empirischen Rekonstruktionen bewähren müssen. Die Entfaltung der Wirkmächtigkeit von Architektur wird an dieser Stelle kurz umrissen. Es werden dabei vier Fragebereiche herausgehoben, die für die Betrachtung von Architektur als handlungsleitende Instanzen von Bedeutung sind. Es sind zum Ersten die Wirkungen 
von Architektur, einerseits in einem physisch-materiellen Sinn und andererseits auch im Sinne der symbolischen Wirkungen mittels an den Gegenstand gekoppelter sozialer Tatsachen, der ,deontischen Macht" (Searle 2012). Zum Zweiten ist die ästhetische Wirkung von Architektur bedeutungsvoll. Vorab ist jedoch zu klären, was Architektur als Artefakt für einen Gegenstand darstellt und damit zusammenhängend die Frage von Gemeinsamkeiten und Unterschieden von Architektur und Kunstwerk.

Architektur ist ein Artefakt. Artefakte sind sozial produzierte Gegenstände. Damit sind sie zum Gegenstand geworden; sie sind zu Natur geronnene Kultur. ${ }^{14}$ Es sind der Kultur äußerliche Objekte, die Träger von sozialen Sinn- und Bedeutungsstrukturen sind.

Eine Besonderheit von Architektur liegt darin, dass sie eine konstituierende Wirkung für eine Gemeinschaft hat; sie ist handlungskonstituierend und sie ist alltagspraktisch handlungsleitend. Letzteres gilt sowohl für die Betrachtung der aggregierten Lebenspraxis Schule als auch für die Angehörigen der Schule, deren Einzelhandlungen durch die Architektur strukturiert werden. Architektur hat noch eine dritte Wirkung, die als außeralltägliche Wirkung beschrieben werden kann, nämlich in ihrer Objektivation als (Kunst-)Werk.

Es können damit drei Bereiche unterschieden werden: Architekturproduktion als soziale Konstruktion einer gesellschaftlichen Praxis, Architektur als handlungsleitende Disposition sowie Architektur als ästhetisches Gebilde und Kunstwerk. Die drei Themenbereiche sind - mit einer gewissen Zurechtbiegung - mit drei Krisen verknüpft, die in dem Theoriegebilde der strukturalen Soziologie und der Methodologie der Objektiven Hermeneutik relevant sind. Sie sollen mit den drei Themenbereichen im Folgenden betrachtet werden: die bereits bekannte Entscheidungskrise (Abb. 1), die Erfahrungskrise sowie die ästhetische Krise oder die Krise durch Muße, die mit der ästhetischen Erfahrung einhergeht.

14 Mit Verweis auf das Prädizierungsmodell von Peirce sind Artefakte zur Substanz gewordene Artifizialität (siehe Oevermann 2000b). 
3.1.7.1 Architekturproduktion als Herstellung praktischer Handlungsdispositionen

Der Beruf des Architekten ist in sich krisenhaft und auf die stellvertretende Krisenbearbeitung gerichtet. Planung besteht darin, die Funktionalität und die Repräsentativität des Bauherrn stellvertretend für diesen zu realisieren. In der Planung werden Tausende von Entscheidungen unterschiedlicher Reichweite getroffen. Die getroffenen Entscheidungen sind äußerst bedeutsam, da Architektur dauerhafte, nur schwer veränderbare Routinen herstellt.

In Bezug auf Architektur sind Entscheidungskrisen vor allem in der Planung und Erstellung von Gebäuden zu thematisieren. Entscheidungskrisen gehen mit stabil-dauerhaftem Anspruch auf Begründbarkeit einher, die durch die Zukunftsoffenheit der Krise aktuell nicht eingelöst werden können, sondern deren Bewährung erst nachträglich überprüft werden kann. In Entscheidungskrisen werden daher langfristige Lösungen gesucht. Für Gebäude gilt dies gesteigert, da Architektur eine Wirkung der longue durée hat. Dies gilt nicht nur für das einzelne architektonische Gebilde, sondern auch für die Konventionen der Architektur, in denen sich die Formsprache, der Stil und die Funktionalität in ihrer historisch-kulturellen Verankerungen repräsentieren. So wird in der Architekturgeschichte jeweils von einer „Grammatik der Anordnungen, von der Stilistik der jeweiligen Funktionalität und der Rhetorik als der Überzeugungskraft“ unterschieden (Barth 2004a: 47). Dieses Wissen ist relativ stabil und wird von den Architekten, von den Bauherrschaften und den Nutzer_innen als Alltagswissen geteilt. Denn als Architekt kann nur erfolgreich sein, wer die Architektursprache spricht, die von der Gesellschaft anerkannt und geteilt wird. Die Konventionen der Architektur stellen eine Form gemeinsamen und damit gesellschaftlich geteilten Wissens dar.

Der Prozess der Entstehung von Architekturen ist für die theoretischen Überlegungen weniger entscheidend. Vielmehr wird dieser für das methodische Vorgehen wesentlich sein, da die Ausdrucksgestalt einer Schulanlage, die oft als historisch gewachsene Gebäudegruppe vorliegt, als Selektion der objektiv vorhandenen Bedeutungsstrukturen in Bezug auf den jeweils vorhandenen sogenannten ,Bestand“ betrachtet wird. An einem Gebäude können jedoch sehr gut die objektiv vorhandenen hypothetischen Möglichkeiten expliziert werden, die 
für den Architekten vorhanden waren und die jeweils unterschiedliche Bedeutungsgehalte verkörpern.

\subsubsection{Architektur als Erzeugung eines Handlungsraums}

Im Unterschied zu Entscheidungskrisen, die zukunftsoffen und in die Zukunft hinein gelöst werden, sind Erfahrungskrisen an die Gegenwärtigkeit im Hier und Jetzt gebunden. Sie sind vom Subjekt losgelöste Erfahrungen, insofern sie äußere Überraschungen sind, die als ,brute fact ' durch ein physisches oder soziales Ereignis eintreten. Bei der Erfahrungskrise muss das Überraschende unmittelbar in Relation zu dem Bekannten gebracht werden. Dieser Abgleich muss richtig integriert und die spontane Reaktion auf ihre Eignung als Krisenlösung bestimmt werden, zum Beispiel wenn aus Unachtsamkeit im letzten Moment einer Wand ausgewichen werden muss.

Die gebaute Umwelt stellt den wesentlichen Bestandteil der Umwelterfahrung dar. Diese Erfahrung ist uns nicht bewusst, sie ist im Körpergedächtnis und im Vorbewusstsein abgespeichert. Trivialerweise beginnt dies mit der Erfahrung, dass Wände hart sind und beim Zusammenprall Schmerzen bereiten. Architektur löst aber auch Leiberfahrungen ${ }^{15}$ aus. Man denke an Gebilde, die explizit für krisenhafte und äußerlich ausgelöste (Selbst-)Erfahrungen gebaut wurden: Balancier- und Klettergeräte, Schaukeln, Rummelplätze und alle anderen Arten von Erlebniswelten, die körper- und leibbasierte Erfahrungen auslösen durch Schaukeln, Rutschen, freien Fall usw. In der Internalisierung körperlicher, krisenhafter Erfahrungen durch architektonische Artefakte werden diese Erlebnisse zu Routinen geformt, die wir mit traumwandlerischer Sicherheit beherrschen bzw. die zum Reflex geworden sind. Die Antizipation der physischmateriellen Gegebenheit erfolgt auf einer Ebene, die Steets (2015: 84 f.) als einen ,präreflexiven Umgang mit Dingen“ bezeichnet. Dieses präreflexive Wissen routinisiert sich in Lernprozessen durch leib- und körperbasierte Erfahrungen, die durch fortlaufende Wiederholungen internalisiert werden.

15 Der Unterschied von Körper- und Leiberfahrung liegt in der Perspektive. Äußere Eindrücke werden als Körpererfahrungen, innere Belange als Leiberfahrungen bezeichnet. 
Gebäude an sich sind keine Überraschungskrisen. Architektur kann aber als potenzielle Erfahrungskrise oder als aus einem ehemaligen brute fact entstandene Routine betrachtet werden, da alltagspraktisch die Routine der Normalfall und die Krise der Grenzfall darstellt. Die Routine, Wänden auszuweichen und Türen zu benutzen, kann zum Beispiel aus Unachtsamkeit jederzeit wieder zu einer schmerzhaften krisenhaften Erfahrung mutieren.

Als physisches Gebilde beziehungsweise Ausdrucksmaterialität, an welche Regeln gebunden sind, hat Architektur primär einen einschränkenden und verhindernden Charakter, der die Bewegungsmöglichkeiten einengt und die Funktionalität einschränkt. Architektur setzt performative Grenzen. Am besten illustriert dies ein Gefängnis. Doch gilt dies generell. Gebaute Räume haben funktional meistens einen funktionalen Bezug. Es sind geplante Räume, die in der Regel in ihren geplanten Intentionen genutzt werden: als Verkehrsflächen, Schlafzimmer beziehungsweise expliziter noch in der Unterscheidung von Eltern- und Kinderschlafzimmer, die wiederum z. B. noch nach Zimmern für das ältere Kind und das jüngere Kind unterschieden werden. Kurz: Räume haben ein (Handlungs-)Programm inne, das wie eine Handlungsanweisung fungiert.

Architektur hat maßgeblich eine steuernde Komponente. Sie ist wesentlicher Bestandteil davon, wie soziale Praktiken oder Bewegungsabläufe rationalisiert und in Handlungsdispositionen übersetzt und eingegossen werden. Dabei stellt Architektur selbstredend auch eine Erweiterung des Handlungsrepertoires und damit der Autonomie dar, indem durch Rationalisierungen der Alltag entlastet wird und Räume für gemeinschaftliche Aktivitäten oder dergleichen entworfen werden. Zudem kann Architektur so ausgestaltet sein, dass sie neue oder differente Handlungsmöglichkeiten zugänglich macht.

Im Zusammenhang mit Schulbauten ist Architektur als Herstellung von Handlungsdispositionen in allen Bereichen involviert. Als ein Beispiel der Aufgabenbewältigung durch Architektur können soziokybernetische Fragen der Steuerung, Disziplinierung und Aufsicht der noch ungestümen Schüler_innen durch Architektur betrachtet werden. So sind auch in räumlich großzügigen Schulgebäuden immer wieder Elemente vorhanden, die etwa vor Ausgängen oder Garderoben künstliche Verengungen erzeugen. Neben den sicherheitsrelevanten Aspekten der Verflüssigung großer Menschenmengen ist der Sinn dieser Verengungen laut Maeder (2008: 10) auch die Aufrechterhaltung einer territo- 
rialen Ordnung, die in der seriellen Ordnung der Zweierreihung als ,normaler Modus der Fortbewegung im Klassenverbund liegt.

Für Kinder ist geradezu konstitutiv, dass sie Dinge vielfältig und multifunktional nutzen, da sie die Handlungsprogramme oder Bedienungsanleitung von Räumen und architektonischen Artefakten (noch) nicht verinnerlicht haben. Kinder nutzen Dinge oft geradezu invers: Sie gehen neben den Gehwegen, klettern auf Mäuerchen und balancieren auf Leitplanken usw. Als ein zentrales Ergebnis dieser Untersuchung zeigt sich, dass architektonische Artefakte in Schulhausarchitekturen in einer spezifischen Weise angeordnet sind. Sie sind so arrangiert, dass nur eine Handlung prädisponiert oder sogar determiniert wird und alle anderen Handlungen verhindert werden. Sie haben damit eine erzieherische Funktion in ihrer disziplinierenden Wirkung, die auf die Internalisierung der ,Handlungsprogramme' gerichtet ist (siehe Kapitel 5.5). Doch soll der Analyse nicht vorgegriffen werden. Als theoretische Explikation ist an diesem Punkt zentral, dass Architektur physisch-materielle Dispositionen schafft, denen Handlungsaufforderungen und -dispositionen eingeschrieben sind. Räume haben ein ,Programm“; sie haben eine spezifische Gestik, die zu gewissen Tätigkeiten einlädt, sie haben auch eine Handlungsdisposition, die gewisse Handlungen erzwingt, die auf die Unterlassung gewisser Praktiken gerichtet ist oder gewisse Praktiken verunmöglicht.

\subsubsection{3 Ästhetische Wirkung von Architektur - Atmosphäre und Kunst}

$\mathrm{Zu}$ den Besonderheiten von Architektur gehört, dass sie sowohl für einen praktischen Nutzen erstellt wird und zugleich eine ästhetische Wirkung hat. Handlungsdispositionen werden nicht nur physisch erzwungen beziehungsweise ermöglicht und suggeriert. Sie werden ebenso durch die ästhetische Gestalt der Architektur und durch symbolisch vermittelte Zeichen erzeugt. Architektur wird dadurch zu einem lesbaren Text im Sinne der Theoriesprache der Objektiven Hermeneutik $^{16}$, der nichtsprachliche Sinn- und Bedeutungsstrukturen darstellt. 
Die Prämisse der Objektiven Hermeneutik ist, dass alle sozialen Ausdrucksgestalten Objektivationen von Sinn- und Bedeutungsstrukturen sind. Sie entstammen menschlichen Handlungen und sind damit Externalisierungen sozialer Praktiken. Besondere nichtsprachliche Ausdrucksgestalten sind dabei ästhetische Gebilde wie Kunstwerke. Sie werden in der qualitativ-rekonstruktionslogischen Forschung auch als Königsweg angesehen. Einerseits da sie als nichtintendierte Daten einer anderen Wahrnehmungsorganisation folgen, als die von Bewusstsein geprägten schriftlichen Überlieferungen. Andererseits sind es ästhetisch verdichtete Wahrnehmungen der Welt. Damit kann über die Analyse von ästhetischen Ausdrucksmaterialitäten eher auf das gesellschaftlich Unbewusste zugegriffen werden.

Architektur gilt als siebte Kunst. Zugleich wird ihr Kunstcharakter auch immer wieder infrage gestellt, da Gebäude nicht primär als Kunstwerke geschaffen werden, sondern als praktische Gegenstände. Ein zweites Merkmal von Architektur ist, dass sie immer gegenwärtig ist. Man kann sich der Architektur nicht entziehen, wie das bei anderen ästhetischen Gebilden möglich ist. Um der Architektur zu entfliehen, bleibt einem nur das Aufsuchen der Wildnis. Diese Feststellung erklärt unter anderem auch die Anziehungskraft der Wildnis als Erfahrungsmöglichkeit des Selbst jenseits der in gebauter Architektur gesellschaftlich präsenten Regeln. ${ }^{17}$

Doch bleiben wir beim Vergleich von Kunstwerk und Architektur: Wie bereits mehrfach erwähnt, ist Architektur ein Artefakt und damit zur Natur sublimierte Kultur. Sie wird im Modus der Perzeption erfahren: Sie wird erlebt, ist aber nicht sprachlich prädiziert. Dies gilt im Prinzip für alle sozial hergestellten Produkte, auch für den Ziegelstein. Die Besonderheit des Kunstwerkes liegt in

die immer nur gelesen werden können. Sinnstrukturen müssen, um wissenschaftlich bearbeitet werden zu können, protokolliert werden. Das Protokoll beschreibt die Objektivation, in welcher der Text festgehalten ist: z. B. das Transkript, der Brief usw.

17 Dies macht die Wildnis zum Sehnsuchts- und Rückzugsort für Systemverweigerer, wie dies in zahlreichen literarischen Verdichtungen (allen voran in Henry David Thoreaus Walden, aber auch in Jon Krakauers Into the Wild, David Gutersons The Other und vielen mehr), aber auch in der Pädagogik am prominentesten bei Rousseau seinen Niederschlag findet. Der Zögling Emil muss den städtischen Raum verlassen, da dieser den ,wahren“ Bildungsprozess verhindert. Erst in der freien Natur ist es möglich, zum ,wahren " Menschsein, der damit also ein kulturloser ist, zu finden (Rousseau 2001). 
der zweckfreien Betrachtung. Beziehungsweise ist der Zweck die sinnliche Erfahrung des Werkes.

Der Künstler, die Künstlerin schafft im Werk eine sinnliche Erfahrung eines vorbewussten ästhetischen Gegenstandes, welcher in eine eigengesetzliche Darstellungsform gebracht wird. Oevermann bezeichnet den Künstler als einen „methodisch kontrollierte[n] Träumer“ (1993: 167). Der Künstler erbringt eine Übersetzungsleistung, indem er seine Erkenntnis als authentische fiktionale Realität in das Werk einarbeitet. Ein gelungenes, für sich autonomes Werk evoziert diese fiktionale Realität aus sich heraus. Sie wird vom Rezipienten nachvollzogen, sofern dieser bereit ist, das Werk zweckfrei auf sich wirken zu lassen. Das heißt, dass das Kunstwerk zweimal erschaffen wird. Einmal vom Künstler und einmal durch den Rezipienten. Das Kunstwerk ,rüttelt' die Erfahrungen wach, indem es eine krisenhafte Erfahrung erzeugt, für die gleichzeitig eine Antwort bereitgestellt wird. Oevermann nennt das Werk „erstarrte Lebendigkeit“" (2000b: 459). Dabei realisiert ein gelungenes Kunstwerk eine Realitätserweiterung gegenüber der Intention der Künstlerin. Kunstwerke verkörpern mehr, als die Künstler_innen bewusst intendieren, indem sie zusätzliche nichtintendierte Erfahrungsgehalte authentisch zum Ausdruck bringen. Dieses Strukturprinzip kann auch für die Architekten angenommen werden. Eine ästhetisch gelungene Architektur ist eine Übersetzungsleistung, in welcher die Funktionalität und die bewohnende Sesshaftigkeit authentisch zum Ausdruck gebracht wird.

Was ist nun an der Rezeption, also der zweiten Erschaffung des Kunstwerks, bezeichnend? Die ästhetische Erfahrung in der ,Krise durch Muße “ ist keine Funktion des Gegenstandes, die alltagspraktisch erfahrbar ist. Im Gegenteil ist für sie entscheidend, dass man vom Alltagsdruck entlastet ist. Ästhetische Erfahrung ist lediglich im Modus der kontemplativen, zweckfreien Perzeption möglich, indem man sich auf eine Interaktion einlässt. Je länger ein Gegenstand um seiner selbst willen betrachtet wird, umso mehr steigt die Wahrscheinlichkeit, in eine krisenhafte Konstellation der Unbestimmtheit zu gelangen und etwas Neues darin zu erfahren. Daher wird die Eigengesetzlichkeit des Kunstwerks nicht nur durch eine Rahmung, z. B. durch den Bilderrahmen oder das Lichterlöschen vor dem Konzert, vom alltagspraktischen Kontext abgehoben. Für die Krise durch Muße ist eine Entkoppelung vom Alltagsdruck konstitutiv. Dies gilt im Prinzip auch für Architektur. Im Modus der ,Krise durch Muße“ kann ein architektonisches Gebilde genau wie ein Kunstwerk zweckfrei betrach- 
tet werden. Oder umgekehrt gilt: Je länger man sich zweckfrei mit einem architektonischen Gebäude befasst, desto größer ist die Chance, zu neuen Erkenntnissen zu gelangen.

Für Architekturen gilt insbesondere der Umstand, dass sie dann ästhetisch als gelungen gelten, wenn sie angeeignet werden können. Denn Architektur hat nicht nur einen künstlerischen Wert, sondern auch - und das ist die maßgebliche Unterscheidung zum reinen Kunstwerk - eine alltagspraktische ästhetische Wirkung. Diese hat natürlich auch das Kunstwerk, in der Wirkung als ornamentaler Schmuck oder als Designobjekt jenseits seiner Funktionen einer potenziellen Erfahrungskonstitution und der Emergenz eines Bildungsprozesses. Bei der Architektur ist die ästhetische Wirkung jenseits des Modus der Erkenntniserfahrung die Entfaltung von Gesten und einer Atmosphäre. Die Atmosphäre wird erzeugt durch den Raum und ist auf ihn bezogen. Loer fasst die Atmosphäre eines Raums äußerst breit:

„Die Totalität aller Handlungsmöglichkeiten, die durch einen Raum eröffnet, angeboten und zu imaginieren auf sinnliche, leiblich spürbare Weise nahegelegt werden, stellt die Atmosphäre dieses Raums dar; zu den imaginierten gehören insbesondere jene Handlungsoptionen, die zu dem und in den Raum passen und ihm verspürt, aber wegen der gegebenen Situation (und der durch sie gegebenen pragmatischen Rahmung) wahrscheinlich nicht realisiert werden." (2013: 73)

Loers Definition bezieht sich auf die Atmosphäre einer ganzen Stadt. Das Konzept kann problemlos auf kleinere Einheiten übertragen werden, wie dies Loer in seinen Beispielen ebenso macht. Als Beispiel wird ein Kaffeehaus angeführt, welches atmosphärisch die Möglichkeit geselliger Interaktion eröffnet. Die Schaffung einer spezifischen Atmosphäre wird nicht ausschließlich, aber zu großen Teilen durch Architektur und andere Artefakte hergestellt, die Handlungsmöglichkeiten eröffnen und einschränken sowie spezifische Handlungsoptionen evozieren. Dies ist nur dann möglich, wenn die atmosphärisch hergestellten Bedeutungen als intuitiv abrufbarer Erfahrungsschatz zum Beispiel in Form von institutionalisierten Tatsachen abgespeichert sind. Durch Atmosphäre ergibt sich eine Verknüpfung von Raum mit institutionalisierten Tatsachen: mit Deutungsmustern, Regeln und Normen, die räumlich gebunden sind beziehungsweise die durch den Raum und ihre Architekturen evoziert werden - denn Atmosphären sind Halbdinge. Sie sind den Subjekten äußerlich. Sie sind mal da, mal nicht, und sie können auf die Subjekte einen Zwang entfalten, z. B. indem sie 
Stimmungen prägen (Steets 2015: 180 f.). Umgekehrt können sie ihre Kraft nur dann entfalten, wenn die Subjekte die Atmosphäre lesen können. Wichtig scheint hier, dass Handlungsdispositionen und die Einschränkung von Handlungsmöglichkeiten durch Architektur nicht nur physisch-materiell, sondern vor allem symbolisch in der Atmosphäre vermittelt werden.

Die Atmosphäre nehmen wir dort besonders auffallend wahr, wo sie außeralltägliche Erfahrungen evoziert. Oft dient sie der Herrschafts- oder Gefolgschaftssicherung: in Kirchen, Schlössern und Prachtbauten ist die Architektur darauf gerichtet, eine Atmosphäre zu entfachen, die Beklemmung, Ehrfurchtsoder auch Glücksgefühle auslöst. Auch Schulbauten schaffen als eigenlogischer Gebäudetyp eine spezifische Atmosphäre, die sowohl in der Außenwirkung als auch in der Innenwirkung wirkmächtig ist. Atmosphäre stellt sich, wie jede ästhetische Erfahrung, erst in der Perzeption beim Rezipienten ein. Zugleich wird sie, analog zum Kunstwerk, über die Objektivierung in einer Ausdrucksmaterialität erzeugt. Daher muss laut Loer die „Untersuchung von Atmosphäre von der konkreten Gestalt und Materialität her entfaltet werden“ (2013: 75).

In Bezug auf den Untersuchungsgegenstand Schulhäuser ist an dieser Stelle ein letzter Punkt anzufügen. Der Künstler wird bei Oevermann nicht nur als methodischer Träumer, sondern im gleichen Satz als „methodisches Kind“ (1993: 167) bezeichnet. Für Kinder ist die ,Krise durch Muße‘ der hauptsächliche Modus der Weltaneignung. Erfahrung konstituiert sich bei Kindern primär in der zweckfreien Betrachtung eines Gegenstandes und damit als ästhetische Erfahrung. Dies gilt umso mehr, je kleiner die Kinder sind. Die ästhetische Erfahrung ist äußerst eng mit dem Bildungsprozess verwoben. Auf die zentrale Rolle der ästhetischen Erfahrung im Bildungsprozess weist auch der Begriff ,Schule“ hin. Etymologisch leitet er sich von dem griechischen Wort scholé ab, was Muße bedeutet. Der Bildungsprozess kann, insbesondere was die Interiorisierung der epistemischen Strukturen betrifft, als sukzessive Ablösung der ästhetischen Erfahrung als alltägliche Erfahrungskonstitution angesehen werden. Die ästhetische Erfahrung wird im Bildungsprozess durch die universellen Regeln des logischen Erschließens, der Formalpragmatik von Sprache und der Moral ersetzt. Dabei ist die Schule als sekundäre Sozialisation ganz wesentlich auf die Internalisierung einer rationalen, sprachlich vermittelten Welterschließung gerichtet. 
Was kann aus diesen Ausführungen über die ästhetische Ausdrucksgestalt von Schulhäusern geschlossen werden? Schulhäuser müssen in ihrer empirischen Materialisierung diesen Aspekt in irgendeiner Art und Weise aufnehmen und realisieren. Dieser Gedankengang soll in die nachfolgenden empirischen Analysen mitgenommen werden.

An dieser Stelle soll eine weitere theoretische und begriffliche Klärung diskutiert werden. Mit der Frage nach der Entfaltung der Wirkung von Architektur ist auch eine der großen Fragen der Soziologie angesprochen: die Frage nach dem Verhältnis von oder der Vermittlung zwischen Struktur und Handlung und damit verbunden auch die Frage, wie Gesellschaft ins Individuum kommt.

Lebenspraxis, egal welches Aggregierungsniveau im Fokus steht, wird in der Methodologie der Objektiven Hermeneutik als Struktur gefasst, welche sich in einem krisenhaften Bildungsprozess als eigengesetzliche Struktur konstituiert und sich weiterhin als autonom handelnde Struktur realisiert. In dieser Betrachtung ist Architektur als Sesshaftigkeit einer lebendigen Handlungs- und Entscheidungsinstanz zu begreifen, die eine eigene Rationalität besitzt. Was Wienke (1999) für die Analyse von Siedlungen annimmt, kann auch auf den Gegenstand einer Gebäudegruppe übertragen werden. Die zentrale Annahme ist, ,dass so etwas wie eine dem Ort innewohnende Logik, eine emergente Rationalität existiert, die nicht von einer einzelnen Bevölkerungsgruppe bewusst geplant wurde, sondern sich über eine langfristige Eigenlogik des Ortes entwickelt“" (Wienke, 1999: 6 f.).

Für die Untersuchung von Architektur ist der Blick darauf zu richten, inwieweit sich die eigenlogische Struktur durch die anfallende Krisenbewältigung in ihrer Eigengesetzlichkeit reproduziert oder transformiert. Eine Strukturtransformation tritt dann auf, wenn bewährte Routinen infrage gestellt werden. Dies kann durch äußere Umstände geschehen, z. B. durch Bevölkerungswachstum oder eine Bildungsexpansion. Es können aber auch von innen motivierte Auslöser sein, wenn das Passungsverhältnis zwischen dem Programm oder der Atmosphäre eines Raums und den Erwartungen an die Praktiken, die in dem Raum stattfinden sollen, divergieren. Wenn dieses Passungsverhältnis unstimmig wird, geraten die raum- beziehungsweise architekturgebundenen Routinen in die Krise, und es wird der Ruf nach neuer, zeitgemäßer Architektur laut. Architektur als Sesshaftigkeit einer lebendigen Handlungs- und Entscheidungsinstanz ist daher nie statisch, auch wenn sie dauerhaft ist. Gebäude stehen in der Gegenwärtigkeit 
unter fortwährendem Bewährungsdruck. Als hypothetische Möglichkeiten von Alternanten, als mögliche Zukunft können sie einer Umwidmung zugeführt werden; sie können um- und angebaut oder erweitert werden, was jeweils einer Strukturtransformation entspricht.

Unter der Betrachtung als Routine werden insbesondere die präformierenden Handlungsmöglichkeiten von Architektur thematisiert. Architektur wurde aus konstitutionstheoretischer Sicht abgeleitet als lang andauernde bewährte Routinen einer kollektiven Praxis. Es sind Objektivierungen des Sozialen, die einen praxisleitenden Charakter haben, in der Herstellung von Handlungsdispositionen über physisch- materielle Dispositionen und über eine ästhetisch hergestellte, symbolvermittelte Atmosphäre. Architektur verkörpert gewisse Normierungen von Abläufen. Mit Löw gesagt, wirkt Architektur als „gebaute Vorstrukturierung von Handlungsvollzügen“ (2007: 81). Diese Vorstrukturierung von Handlungsvollzügen können in der Betrachtung als bedeutungsgenerierende Regeln im Parameter I auch als soziale Konstruktionen gesehen werden (siehe Maiwald 2013). Wenn Architektur als „sinnhafter Aufbau gebauter Welt“ (Steets 2015) betrachtet wird, können deterministische und nichtdeterministische Wirkungen von Architektur unterschieden werden. Als deterministische Wirkung verhindert Architektur bestimmte Handlungsmöglichkeiten; sie schränkt Performanz ein, indem physische Bewegungen, Ansammlungen, Einblicke usw. unterbunden werden. Als nichtdeterministische Wirkung entwirft und präformiert sie Handlungsmöglichkeiten.

Damit ist Architektur ein Erzeugungsprinzip gebauter Bedeutungsstrukturen; insbesondere natürlich für diejenigen Bedeutungen und Funktionen, für die sie gebaut ist und deren Sinnstruktur sie explizit verkörpert: zum Beispiel als Turnhalle, Garderobe oder Klassenzimmer. Sie erzeugt Regeln für die Praktiken, für die sie idealtypisch geplant und erstellt worden ist. Als realisierte Sinnstruktur erzeugt Architektur aber auch nichtintendierte und/oder alternante Möglichkeiten. Die Eigenschaften werden nicht von den Gebäuden determiniert, sondern sie sind von den Praktiken abhängig, die in ihnen vollzogen werden, und der Art, wie mit der Architektur interagiert wird. Architektur wirkt dabei als Einflussstruktur, welche den Möglichkeitsraum der Praktiken erzeugt.

Als konstitutionstheoretische Prämisse wurde vorausgesetzt, dass Gebäude und Objekte die Macht besitzen, unsere Handlungen zu steuern, aber auch gewisse Gefühle wie Ehrfurcht oder Furcht auszulösen. Gebäude stellen institutio- 
nalisierte Tatsachen dar. Es soll daher abschließend skizziert werden, wie die sozialen Regeln an Gegenstände gekoppelt werden, so dass diese zu institutionalisierten Tatsachen werden.

\subsubsection{Architekturen als Objektivationen institutionalisierter Regeln}

Gegenstände - und Personen - entfalten Macht. Schüler_innen gehorchen der Schulglocke; sie befolgen gezogene Grenzen auf dem Pausenhof, wo Fußball gespielt werden darf und wo nicht, sie akzeptieren, dass sie nur zu bestimmten Zeiten gewisse Räume betreten dürfen usw. Dinge und Räume haben eine Wirkung, indem institutionalisierte Regeln an sie gebunden sind.

Eine Theorie, die eine dauerhafte und stabile Anbindung von institutionalisierten Tatsachen an Gegenstände oder Personen erklärt, findet sich bei Searle (1996, 2012). Die Theorie erklärt, wie Dingen und Personen ein Status zugeschrieben wird, der mit einer Funktion verbunden ist und ihnen Macht verleiht.

\footnotetext{
„Die maßgebliche Rolle gesellschaftlicher Institutionen und der Zweck ihrer Existenz ist nicht, die Menschen im eigentlichen Sinne einzuschränken, sondern vielmehr, neue Formen von Machtbeziehungen zu schaffen. Die Institutionen der Menschen haben vor allem die Funktion, zu etwas zu befähigen, da sie Machtpositionen schaffen - aber eine spezielle Form von Macht. Macht ist gekennzeichnet durch Begriffe wie Rechte, Pflichten, Verpflichtungen, Befugnisse, Erlaubnisse, Ermächtigungen, Anforderungen und Zertifizierungen. Ich bezeichne alle als deontische Macht." (Searle 2015: 115)
}

Deontische Macht meint bei Searle eine stabile Verknüpfung von Routinen wie Rechte und Pflichten, d. h. dass Verhaltensaufforderungen an Dinge und Personen gebunden werden. Generell können zwei Formen von Regeln unterschieden werden (Searle 2012: 22 f.): Es gibt Regeln, die regulierend wirken, und es gibt konstitutive Regeln. Regulierende Verhaltensweisen haben die Form einer Verhaltensaufforderung: ,Mach das!‘. Es sind z. B. die oben explizierten Handlungsdispositionen des Unterrichtsbeginns durch das Klingeln der Schulglocke. Konstitutive Regeln, wie zum Beispiel die Spielregeln eines Schachspiels, konstituieren ein eigenlogisches Gebilde, welche das Spiel ausmachen. Die konstitutiven Regeln verlangen danach, dass die regulierenden Regeln an Dinge und Objekte stabil gekoppelt sind: Dinge (und Menschen) repräsentieren einen Status bzw. sie haben eine Statusfunktion. Dabei muss, ,um überhaupt zu existie- 
ren, (...) eine Statusfunktion als existent repräsentiert werden. Sprache oder irgendeine Form von Symbolik stellen dabei das Mittel zur Repräsentation dar" (Searle 2015: 117). Regeln, die mit Grenzen einhergehen, werden zum Beispiel mittels architektonischer Artefakte repräsentiert: Dies gilt für Schulanlagen, die oft mit Einfriedungen oder anderweitig markiert sind, aber auch das Betreten gewisser Räume. Im Klassenzimmer, in der Garderobe oder im Lehrerzimmer sind jeweils spezifische Regeln gültig, und das Betreten dieser Räume geht mit Statusverschiebung bzw. -übernahmen einher.

Dabei ist festzuhalten, dass die Regeln objektiv d. h. unabhängig von der subjektiven Anwendung gültig sind. Auch wenn ich nach dem Klingeln auf dem Pausenhof verbliebe, hat die Klingel deontische Macht inne. Die an sie geknüpften Verhaltensaufforderungen und -verbote behalten ihre allgemeine Gültigkeit, da sie kollektiv anerkannt werden (und unter Umständen bei Nichtbefolgung Sanktionen nach sich ziehen). Dies ist der Fall, da sie eben als institutionalisierte Regeln an den Statusindikator ,Schulglocke“ gebunden sind. Es sind also objektive, allgemeingültige Regeln, die das Individuum von Handlungsentscheidungen entlasten und für das Individuum wie normative, zu befolgende Handlungsgebote wirken.

Methodisch und methodologisch gesehen ist der Regelbegriff in der Objektiven Hermeneutik nicht determinierend zu verstehen, sondern als bedeutungserzeugend. Eine Regel bestimmt nicht, was gemacht wird. Eine Regel erklärt vielmehr, welche Bedeutung der Vollzug einer Handlung hat; also was es heißt, etwas zu tun. Dabei ist anzufügen, dass Regeln und Struktur nur analytisch getrennt werden können. In ihrem Wesen sind sie eine unauflösbare Einheit. Regeln objektivieren sich nie an sich. Die Regeln objektivieren sich in Ausdrucksgestalten zum Beispiel eben in Architekturen oder anderen Artefakten. Die Ausdrucksgestalten können sinnlich wahrgenommen werden, nicht aber die Regeln an sich. Diese können lediglich am Gegenstand rekonstruiert werden, zum Beispiel eben am Klingeln der Schulglocke, welche die Regeln eines zeitlich und räumlich normierten Unterrichtes in einem spezifischen Zeitgefäss der Lektionen in ihrem Beginn und Ende markiert.

Es ist anzunehmen, dass Schulbauten sozialisations- und gesellschaftstheoretisch gesehen besondere Bedeutung zukommt. Sie stellen nicht einfach weitere Gebäude in einer gebauten Umwelt dar. Sie sind als Bauten konzipiert, deren ,Programm‘ Sozialisation und Bildung beinhaltet. Damit bilden sie im Gegen- 
satz zur naturwüchsigen primären Sozialisation die Verkörperung der Institution der sekundären Sozialisation ab. Es kann hypothetisch angenommen werden, dass sie die gesellschaftlichen Regeln besonders zugespitzt repräsentieren müssen, da diese in ihnen internalisiert werden müssen.

\subsubsection{Architektur als handlungsleitende Routinen und Krisenevozierung}

Architektur zu untersuchen, ist anspruchsvoll, da sie ihre Effektivität perzeptuell in einem nichtsprachlichen Bereich entfaltet, der reflexiv nicht direkt zugänglich ist. Zudem wirkt sie auf unterschiedlichen Ebenen. Sie hat eine konstituierende Funktion, sie hat eine alltagspraktisch-handlungsleitende Funktion wie auch eine außeralltägliche Funktion als Kunstwerk. Umgekehrt kann forschungspraktisch konstatiert werden, dass Architekturen aus diesen Gründen ein außerordentlich dichtes, authentisches und vielseitig zu befragendes Datenmaterial darstellen. Architektur sollte gerade daher eine einträgliche Fundgrube für die Analyse des gesellschaftlich Bewussten und Unbewussten sein. Zudem erlaubt es die architektursoziologische Herangehensweise, Akteure und Strukturen in einer verbundenen Perspektive zu sehen.

Ziel dieses Kapitels war, über konstitutionstheoretische Fragen von Architektur die Grundsteinlegung für das Forschungsvorhaben und die anschließenden Analysen zu legen. Es ist das Fundament des Forschungsvorhabens. ${ }^{18}$

Das Fundament besteht aus den Bausteinen der strukturalen Soziologie, insbesondere den Prämissen humaner Lebenspraxis, die sich krisenhaft konstituiert. Ebenso wichtig erscheint mir ihre Verankerung in der Theorie der sozialen Raum-Zeitlichkeit. Damit können Gebäude als Sesshaftigkeit einer kollektivgemeinschaftlichen Lebenspraxis bestimmt werden. Gebäude sind als Behausungen die Innen-Außen-Abgrenzung der ,bewohnenden ‘ Lebenspraxis. Sie sind Objektivationen dieser Sesshaftigkeit. Als verkörperte Sesshaftigkeit muss die Architektur die Funktionalität der Praktiken der Lebenspraxis als symbolisch vermittelte Repräsentation nach außen darstellen.

18 In der Genese war der Vorgang oft umgekehrt. Vieles, was hier darstellerisch vorweggenommen wird, hat sich aus den Rekonstruktionen in den empirischen Analysen ergeben. 
Damit sind schon die ersten sichtbaren Elemente benannt, die aus dem Fundament ragen und das Gerüst dieser Untersuchung darstellen. Architektur als gemeinschaftliche Sinnstruktur muss das gesellschaftliche Wissen, die gesellschaftlich relevanten Regeln und Normen in den Konventionen der gemeinschaftlichen Architektursprache interpretieren, damit sie von den Einzelsubjekten erfahren und gelesen werden können. Damit stellt die Architektur das „Gesicht der Gesellschaft“ (Castoriadis, zit. nach Delitz, 2010) dar, das es ihr erlaubt, sich zu erkennen.

Das Gesicht der Architektur, als Einzelobjekt und als gesellschaftliche Konvention der Ausdrucksform, wird nicht im Modus der kognitiven Erkenntnis, sondern über Perzeption als Erfahrungsgehalte im kollektiven Gedächtnis und im Leibgedächtnis abgespeichert. Architektur nimmt sowohl für die Stabilisierung als auch für die Transformation sozialer Strukturen eine zentrale Rolle ein: Sie ist eine Objektivierung der sozialen Ordnungsstruktur (über die Repräsentation) und der sozialen Praktiken (über die Funktionalität) in Abhängigkeit von den technologischen, ökonomischen und kulturellen Potenzialen und Möglichkeiten einer Gesellschaft. Das heißt, Gesellschaftsformen sind mit Architekturformen unzertrennlich verwoben.

Der zweite Teil des Kapitels zielte auf die Frage der Wirkungsweisen und Wirkmächtigkeiten der Architektur für die Lebenspraxen in den Gebäuden. Als äußerst dauerhafte, krisenhaft produzierte Gebilde fungieren Architekturen alltagspraktisch als Routinen. Architektur schränkt Performanz ein und schafft spezifische Handlungsoptionen und -anleitungen, indem sie räumlich gebundene Handlungsdispositionen physisch-materiell und symbolisch realisiert.

\subsection{Der Auftrag von Schulgebäuden - Orte gesellschaftlich organisierter Sozialisationsprozesse}

Jedes Gebäude hat einen Auftrag: Schulhäuser sind dafür gebaut, Schulen zu beherbergen. Doch wofür stehen Schulen? Aus einer weit gefassten makrosoziologischen Perspektive ist der Auftrag der Schule die - anteilmäßige - Leistung zur Reproduktion der Gesellschaft über die Sozialisation der Individuen. Dies verweist auf einen Doppelcharakter, der der Sozialisation innewohnt: Einerseits stellt sie die Reproduktion der Gesellschaft sicher und hat einen kollektiven 
Charakter. Andererseits ist die Reproduktion der Gesellschaft nur indirekt möglich, indem die Individuen zu autonomen, handlungsfähigen Subjekten sozialisiert werden. Das bedeutet, dass jede Gesellschaft Institutionen ausbilden muss, die auf die Erreichung der Handlungsfähigkeit der Subjekte als vollwertige Mitglieder der Gesellschaft gerichtet sind. Dies ist nur über die Ausbildung und Sicherung der Autonomie des Einzelnen möglich, wobei die Autonomie in Abhängigkeit des Autonomiepotenzials einer jeweiligen Gesellschaft zu sehen ist. Nach Oevermann (2012: 178) ist dies ,ein universelles Problem der Gattung Mensch und ein allgemeines Systemproblem der Gesellschaft“.

Die realisierte Form der Institutionalisierung wird an den konkreten Fällen in den Fallrekonstruktionen erschlossen. Der nachfolgende Teil der theoretischen Bestimmungen wird deshalb äußerst kurz gehalten. Es sind aber notwendigerweise einige Eckpfeiler einzuschlagen, die den Sozialisations- und Bildungsprozess konturieren. Damit soll programmatisch entworfen werden, was von der Analyse von Schulbauten erwartet werden kann.

\subsubsection{Zur Struktur von Sozialisations-, Bildungs- und Lernprozessen}

In der Summe wird ein sozialisiertes Subjekt als eine individuierte Lebenseinheit bezeichnet,

„die der logischen und moralischen Urteilsfähigkeit, des kumulativen Lernens und synthetischen Erfahrungsurteils, der Selbstreflexion und Normenkritik, der Artikulation eigener Bedürfnisse, des strategischen Handelns und des adäquaten Ausdrucks unmittelbarer Affektionen fähig ist." (Oevermann 2012: 180)

Unter Sozialisation wird die Entwicklung verstanden, wie die Subjekte zu handlungs- und damit gesellschaftsfähigen Subjekten werden. Dabei wirkt Sozialisation dem angeborenen Egozentrismus des Kindes entgegen. Es ist ein krisenhafter Prozess, der eine Anpassung an die Welt erfordert, indem es dem Kinde gelingen muss, ,aus seinem Ich herauszutreten, um sich eine objektive Vorstellung der Wirklichkeit zu konstruieren“ (Piaget 2017: 57). Gleichzeitig muss sich das Kind diese ,objektive ${ }^{`}$ Welt zu eigen machen, indem es sich das gesell- 
schaftliche Wissen und die sozialen Regeln aneignet und internalisiert und gleichzeitig die epistemischen Strukturen entwickelt bzw. interiorisiert. ${ }^{19}$

Im Modell der Lebenspraxis wurde ausgeführt, dass sich Erfahrungen Bildungsprozesse sind nichts anderes als Erfahrungsprozesse - krisenhaft entwickeln und nie in Form von Routinen. Die Sozialisanden internalisieren Erkenntnisse dann, wenn sich in krisenhaften Erfahrungsprozessen ein Wissen oder eine Fertigkeit als erfolgreiche Bewältigung einer Krise bewährt und damit als Routine abgespeichert wird, auf die zukünftig zurückgegriffen werden kann. Routinen sind daher - oft unbewusste, habitualisierte - Ausführungen von ehemals in Krisen angeeigneten Erfahrungen. Wie eignen sich die Sozialisanden Wissen und insbesondere auch Regelwissen an? Es sind zwei Arten von Sozialisationsprozessen zu unterscheiden: naturwüchsige, ungesteuerte Sozialisationsprozesse, die sowohl Bildungs- als auch Lernprozesse beinhalten, und pädagogische Prozesse, die gesteuerte und intendierte Prozesse sind.

Die Prozesslogik naturwüchsiger, sozialisatorischer Interaktion beschreiben Garz und Raven (2015: 67 f.) in fünf Schritten anhand des Beispiels einer Eltern-Kind-Interaktion. Erwachsene geben dem Kind in der Interaktion einen Sinnüberschuss gegenüber dem faktischen, kognitiven, sprachlichen und mentalen Entwicklungsstand des Kindes mit, indem sie so interagieren, als ob das Kind das schon verstehen könnte. Die Erfahrungs- und Sinnüberschüsse werden als Erinnerungen abgespeichert und sind die Voraussetzung für eine nachträgliche Erschließung der Bedeutungsgehalte. Das von den Erwachsenen vorgegebene Erfahrungsmaterial fungiert für das Kind wie eine stellvertretende Handlung und Deutung durch die Eltern. Das ist daran ersichtlich, dass die Nachahmung für Kinder ein zentraler Modus der Welterfahrung und Quelle von Bildungsprozessen ist. Dabei machen sie neue Erfahrungen und gelangen zu neuen Erkenntnissen, indem das Kind

19 Für den Sozialisationsprozess unterscheidet die strukturale Soziologie die Aneignung und Verinnerlichung von Inhalten wie Wissen, soziale Normen und Regeln (Internalisierung) und die Verinnerlichung von epistemischen Strukturen (Interiorisierung); die formal logischen kognitiven Kompetenzen, die moralische Urteilskraft und die Sprachkompetenzen, die in der Ontogenese des Subjektes entwickelt werden. 
,auf die entsprechend seines Entwicklungsstandes noch nicht oder nur partiell begreifbaren Handlungen bzw. Deutungen (...) [zurückgreift] und versucht, den objektiven latenten Sinn der ursprünglichen Interaktion (...) rekonstruktiv zu begreifen. Dabei vollzieht es eine eigentätige Überführung von objektiv latentem Sinn in subjektiv intentionalen Sinn“ (Garz/Raven 2015: 67 f.).

Damit eignet sich das Kind sukzessive neues Wissen an und verinnerlicht gleichzeitig die gesellschaftlichen Regeln und Normen, nach denen die Interaktionen strukturiert sind. Zugleich wirken die Internalisierungen auch auf die Entwicklung der epistemischen Strukturen ein, was eine Transformation der Interpretationskapazität hin zu einem höheren Niveau zur Folge hat. Der Bildungsprozess ist damit ein andauernder Strukturtransformationsprozess.

Was am Beispiel der naturwüchsigen Eltern-Kind-Interaktion ausgeführt wurde, kann auch für die Aneignungsformen der ,gebauten Welt' angenommen werden. In dem naturwüchsigen Umgang mit architektonischen Artefakten routinisieren sich Erfahrungsgehalte und werden internalisiert und habitualisiert. Dabei ist anzunehmen, dass die Internalisierung der Welt, was in der modernen urbanen Gesellschaft immer die ,gebaute Welt ${ }^{\dagger}$ ist, sehr früh, wahrscheinlich schon im Mutterleib beginnt. In einer Analogie zur Erwachsenen-KindInteraktion, in der jeweils Erwachsene ganz naturwüchsig eine Sprache sprechen, die den Wahrnehmungskapazitäten der kindlichen Welt entspricht, wirkt die Architektur im Prinzip unvermittelt in ihrer ganzen Macht. Sie ist als Umwelt Interaktionsobjekt, in welcher neue Erfahrungen gemacht werden, die ihrerseits wiederum als Strukturtransformationen auf den Entwicklungsstand der Raumwahrnehmung und auf die Ausbildung von Handlungskompetenzen und Raumwissen wirken (vgl. dazu Piaget und Inelder 1999). Doch soll vorderhand noch auf die Spezifika schulischer Interaktionen eingegangen werden, die sich von den naturwüchsigen Interaktionen in der Primärsozialisation unterscheiden.

Unterricht unterscheidet sich von naturwüchsigen Interaktionen, indem er stark formalisiert ist. Die Besonderheit liegt darin, dass der Lerngegenstand von der Lehrperson induziert und/oder evoziert wird. Im Gegensatz zu den naturwüchsigen sozialisatorischen Interaktionen, die von der Neugierde des Kindes geprägt und von diesem oft fragend eröffnet werden, liegt das pädagogischdidaktische Vorgehen darin, dass die Bildungs- und Lernprozesse von der Lehrperson ausgewählt und entworfen werden.

Lernprozesse werden begrifflich eingeschränkt verstanden als Prozesse einer intendierten Wissensvermittlung, die innerhalb von arrangierten Lehr-Lern- 
Settings stattfinden. Die Lehrperson entwirft eine Problematik, die für die Schüler_innen Neuland - also krisenhaft - ist, wobei gleichzeitig didaktisch aufbereitete Lösungsangebote oder -wege unterbreitet werden. Die pädagogische Aufgabe ist die Initiierung von Bildungs- und Lernprozessen. Dies ist die Grundstruktur pädagogischen Handelns, zumindest in einer professionalisierungstheoretischen und zu weiten Teilen auch in einer erziehungswissenschaftlichen Perspektive auf Schule, welche die didaktisch angeleitete Emergenz von Bildung und Erziehung fokussieren.

Bildungsprozesse verändern das Subjekt, da es gebildet wird. Es durchläuft also eine Strukturtransformation, welche das Welt- und Selbstverständnis des Kindes transformieren. Lernprozesse meinen das Aneignen und Abspeichern von Wissen, Fähigkeiten und Fertigkeiten, also die Internalisierung von Routinen, ohne dass damit zwingend ein Bildungsprozess des Verstehens einhergeht. Lernprozesse können Bildungsprozesse initiieren oder mit ihnen einhergehen. Umgekehrt sind Bildungsprozesse immer auch Lernprozesse, und sie bedingen auch Lernprozesse.

Empirische Unterrichtsanalysen stellen immer wieder fest, dass im Unterricht Lernprozesse überdominant und Bildungsprozesse nur in Ausnahmefällen anzutreffen sind (zum Beispiel Combe/Helsper 1994; Franzmann/Pawlytta 2005; Gruschka 2011, Wernet 2003). Mehan (1979) stellt in einer schulethnographischen Untersuchung fest, dass die Interaktionen im Unterricht in großer Mehrheit einem dreischrittigen Interaktionsschema entsprechen, das auf die Internalisierung von Routinen gerichtet ist: Eröffnet wird die Interaktion häufig durch einen Initiierungsakt der Lehrperson in Form einer Frage. Auf diese folgt ein Antwortakt einer Schülerin, eines Schülers. Das dritte Element beschreibt Mehan als evaluativen Akt, in dem die Schülerantwort von der Lehrperson kommentiert wird. Wenzl (2014) kommt zu einem ähnlichen Ergebnis und nennt die von Mehan analysierte Interaktionsordnung die „Elementarstruktur unterrichtlicher Interaktion“ (2014). Darauf aufbauend untersucht Wenzl Interaktionen, die nicht diesem Muster folgen und die potenziell krisenhaft sind. Er kommt zum Schluss, dass in den Interaktionen, die von Schüler_innen initiiert werden, die Antworten der Lehrpersonen eher darauf gerichtet sind, die Verletzung der „Elementarstruktur“ zu ,reparieren“, als dass auf die Fragen der Schüler_innen eingegangen wird. Wenzl interpretiert die empirisch anzutreffende Dominanz von Lernprozessen in Fortsetzung von Parsons (2012) und Wernet 
(2003) aus der Notwendigkeit der Einsozialisierung von Rollenförmigkeit durch die Schule.

Schule hat in dieser Perspektive vor allem eine erzieherische Funktion. Unter Erziehungsprozessen sollen Prozesse verstanden werden, die wie Lernprozesse strukturiert sind, deren Fokus jedoch nicht die Wissensaneignung, sondern das Verhalten und damit die Internalisierung von Normen sind. Erziehungsakte sind intentionale Akte des Erziehenden. Sie enthalten eine Willensbildung des Erziehenden, eine Zielfokussierung und die Planung von Erziehungsschritten und

-mitteln. Dabei ist für die Schule wesentlich, dass sie als Institution erzieht. Die Schule ist in der modernen Gesellschaft die zentrale Sozialisationsinstanz, die die als universell geltenden gesellschaftlichen Regeln und die Entwicklung rollenförmigen, d. h. gesellschaftlichen, Handelns im wesentlichen einsozialisiert. Sie unterscheidet sich damit konstitutiv von der familialen Sozialisation.

Um diese zwei Sozialisationsinstanzen noch besser zu fassen, sollen im Folgenden die unterschiedlichen sozialisatorischen Komponenten von Primärund Sekundärsozialisation ausgeführt werden.

\subsubsection{Strukturvarianten sozialisatorischer Interaktion}

Nach Helsper (2012: 454) ist Sozialisation als jener ,in sozialer Interaktion gründende Prozess zu rekonstruieren, in dem über strukturale Krisen der Individuation lebenspraktische Autonomie in verschiedenen Strukturvarianten generiert wird“. Diese Strukturvarianten sollen im Folgenden umrissen werden, da sie für den Untersuchungsgegenstand der Sekundärsozialisation durch die Schule relevant sind.

Aus einer strukturtheoretischen und zeitdiagnostischen Perspektive kann die Sozialisation dahingehend unterschieden werden, ob sie eingebettet in eine generationale Ordnung zwischen Erwachsenen und Sozialisanden oder ob sie zwischen Gleichaltrigen in der Peer Group vonstattengeht. Zudem kann unterschieden werden, ob die Sozialisation in einer naturwüchsigen sozialisatorischen Interaktion stattfindet oder ob sie innerhalb einer organisierten und arrangierten Bildungsangelegenheit vorliegt, die sich durch pädagogisch-intendierte Lern-, Erziehungs- und Bildungsprozesse auszeichnet. Damit können drei Varianten 
von Sozialisationsbereichen unterschieden werden, die je eigenen spezifischen Gesetzlichkeiten folgen und die von unterschiedlichen Handlungsorientierungen geprägt sind. In idealisierter Form sind dies: zum Ersten die Familie als Ort der naturwüchsigen Primärsozialisation innerhalb der generationalen Ordnung wie auch innerhalb der Geschwisterreihenfolge. Zum Zweiten ist dies die Peer Group als Bereich sozialisatorischer Interaktion unter Gleichaltrigen. Zum Dritten ist dies die Schule als Ort pädagogisch intendierter Prozesse sekundärer Sozialisation. Diese drei Bereiche sollen im Folgenden ausgeführt werden.

Die Primärsozialisation in der Familie ist in einer strukturfunktionalistischen und strukturalen Perspektive durch die diffusen Beziehungen zwischen ganzen Personen bestimmt (vgl. Parsons 1968; Wagner 2001). Dafür ist eine reziproke, affektive Basis konstitutiv. Zudem sind diffuse Beziehungen unkündbar: Die Personen sind nicht substituierbar. Eltern können nicht ausgewechselt werden beziehungsweise sie sind auch dort, wo sie aus irgendwelchen Gründen absent sind, in ihrer Abwesenheit allgegenwärtig und lebensbestimmend. Im Weiteren sind die diffusen Beziehungen durch eine Allzuständigkeit bestimmt. Sie beruhen auf einem bedingungslosen Vertrauen und sind in irgendeiner Form körperbasiert. Dies hat zur Folge, dass das Gegenüber als ganze Person in seiner Liebenswürdigkeit inklusive seiner Mängel und Mäkel angenommen wird. Als Sozialisations- und Bildungsinstanz ist die Familie vor allem in ihrer Naturwüchsigkeit der sozialisatorischen Interaktion relevant.

Der zweite sozialisatorische Bereich sind die Beziehungen unter Gleichaltrigen. Sie sind für die Entwicklung kooperativ-symmetrischer Kommunikation und moralischer Strukturen, insbesondere für die demokratische Vergemeinschaftung, wesentlich. Eine solche kann nur mit Bezugspersonen außerhalb der Familie eingeübt werden (Oevermann 2009b). Zudem sind diese Beziehungen symmetrisch-reziprok, im Gegensatz zu den generationalen Beziehungen, die durch Asymmetrie gekennzeichnet sind (siehe ausführlich Krappmann/Oswald 1995).

„Für die schulischen Zusammenhänge ergibt sich daraus eine doppelte Bedeutung der Peers: Zum einen stellen sie - als zentraler Adressat des pädagogischen Arbeitsbündnisses - eine «Neugierigen-Gemeinschaft» dar, in der die gegenseitige Unterstützung im Sinne einer «tutorialen Lernkultur» (Oevermann 1996: 176) zentral ist. Zum anderen bilden sie innerhalb des schulischen und auch außerschulischen Zusammenhangs eine eigenständige Peerkultur, der die 
wichtige sozialisatorische Bedeutung zukommt, «den Schülern gerade auch im Konflikt mit den Lehrern und in der Auflehnung gegen sie» (ebd.: 147) einen Zuwachs an Autonomie und Einzigartigkeit zu eröffnen. Dies wird über die Sozialisationsinstanz der Schule hinaus auf die generelle Bedeutung der jugendlichen Peer-Vergemeinschaftung für die vierte Individuationskrise der Adoleszenz bezogen." (Helsper 2012: 459, Hervorhebungen im Original)

Damit sind zwei Perspektiven auf die Peer Group angesprochen, die auch für das Untersuchungsvorhaben von Bedeutung sind. Es ist festzuhalten, dass Schulen den räumlichen Ort darstellen, wo Kinder sich kennenlernen und treffen. In dem relativ stabilen Klassenverbund konstituiert sich eine für die Sozialisation relevante Peer Group. Dieser Bezug zur Schule als räumliche Instanz ist nicht nur in Bezug auf Unterricht zu sehen. Unter den Bedingungen der „Verinselung“ und „Zonierungen“ kindlicher Lebensräume (Kogler 2015) haben Schulanlagen für Kinder auch außerhalb von Unterrichtszeiten eine wichtige Bedeutung als Treffpunkte und Freizeiträume. Insbesondere in heutigen, urban verdichteten Räumen bleiben Schulen als residuale Räume übrig, in denen sich Kinder überhaupt außerhalb der Kontrolle von Erwachsenen aufhalten können. Damit sind Schulen nicht nur als Orte geplanter und formeller Bildungsveranstaltungen relevant, für die sie primär konzipiert sind, sondern auch für naturwüchsige Bildungsgelegenheiten in der Peer Group. Räumlich gesehen sind diese beiden Sphären in Schulanlagen relativ strikt geschieden: das Schulhaus als Ort des Unterrichts und der Schulhof als Ort des Nichtunterrichts.

Der dritte sozialisatorisch relevante Bereich ist die sekundäre Sozialisationsinstanz: die Schule. Sie stellt den staatlich organisierten und institutionalisierten Lernort für die Bildung und Sozialisation von Kindern dar. Sie ist ein Phänomen der Moderne und auch eng mit der Entwicklung des Nationalstaates verbunden. Der Kern von Schule bildet die Unterrichtung von Kindern, welche der Vermittlung eines spezifisch kodierten, expliziten Wissens in einem spezifischen Setting der Schulförmigkeit in Prozessen didaktisch-pädagogischer Praktiken dient. Schulen sind explizite Orte des formellen Lernens, im Gegensatz zur Familie oder zur Peer Group, die als Orte des informellen und non-formalen Lernens zu verstehen sind.

Wesentlich ist, dass alle drei Beziehungsvarianten in der Summe ihren spezifischen Anteil in der Ontogenese und der Sozialisation zum praxisfähigen Subjekt leisten (vgl. Garz/Raven 2015; Oevermann 2012). Sie sind nicht substi- 
tuierbar. Das heißt, wenn einer der Bereiche wegfällt, ist die Sozialisation und der autonome Vollzug von Lebenspraxis für das konkret betroffene Subjekt unter Umständen wesentlich erschwert.

Schulen als Sozialisationsorte, und damit auch Schulhäuser und -anlagen, sind in den zwei letztgenannten Sphären von großer Bedeutung. Die Familie und die Schule werden in allen Sozialisationstheorien als zu unterscheidende Bereiche aufgefasst, die sich als konträre, je nach Ansicht harmonisch ergänzenden oder tendenziell konfligierenden Bereiche komplementieren (siehe zum Beispiel Luhmann 1988; Parsons 1968; Tyrell 1985).

Die schulische Sozialisationsfunktion besteht für Parsons (1968) im Wesentlichen darin, bei den Sozialisanden Bereitschaften und Fähigkeiten auszubilden, die für die Handlungsfähigkeit unabdingbar sind. Parsons stellt zwei motivationale Bereitschaften ins Zentrum: die Bereitschaft zur „Verwirklichung der allgemeinen Werte der Gesellschaft“ und die Bereitschaft „zur Erfüllung eines spezifischen Rollentyps innerhalb der Struktur der Gesellschaft" (Parsons 1968: 162). Mit diesen Bereitschaften sind zwei Fähigkeiten verknüpft: einerseits die Fähigkeiten, Kompetenzen und Fertigkeiten zu erlangen, die dazu dienen, eine bestimmte Rolle auszuüben, z. B. die Rolle eines Verkäufers oder einer Dozentin. Und andererseits die Internalisierung einer „Rollenverantwortlichkeit“. Das sozialisierte Subjekt verhält sich rollenkonform, also so, wie das von den anderen aufgrund der eingenommenen Rolle erwartet wird. Das zentrale Moment schulischer Sozialisation ist bei Parsons die Anpassung an die gesellschaftlichen Anforderungen durch die Subjekte, die darin liegen, den rollenförmigen, interpersonalen Erwartungen zu entsprechen.

Worin besteht Rollenförmigkeit, und wie wird sie internalisiert? Unter Rollenhandeln wird verstanden, dass die Handlungskoordination und das Interaktionsverhalten des Zusammenlebens über eine Einschränkung der Verhaltensorientierung gesteuert werden. Dies wird durch eine rollenförmige Adressierung der Schüler_innen einsozialisiert. Die Interaktionen in der Schule zwischen Professionellen und Schüler_innen unterscheiden sich dadurch grundsätzlich von denjenigen der familiären Interaktion, dass sie nicht auf einer partikularen, diffus-affektiven Basis stehen, sondern dass wesentliche Teile der Beziehung konträr zu den Orientierungen in der Familie geregelt sind: Sie sind streng rollenförmig. Das beginnt damit, dass das Personal der Schule unpersönlich und ersetzbar ist. Die Individuen werden aufgrund von Leistungen und affektiv neut- 
ral adressiert. Die Beziehungen zwischen Lehrpersonen und Schüler_innen sind spezifisch auf definierte Fokusse eingeengt. Während Schüler_innen diffus agieren, ${ }^{20}$ müssen die Lehrpersonen auf einer strikten Einhaltung der Spezifität der Beziehung beharren. Indem die Lehrpersonen selbst strikt rollenförmig agieren, verinnerlichen die Schüler_innen ,das reziproke Muster der Rollenbeziehungen“ (Parsons 1968: 175). Für die Herausbildung der gesellschaftlich notwendigen Rollenförmigkeit der Sozialisanden ist es Aufgabe der Schule, strikt rollenförmig zu agieren. Oder anders gesagt: Die Art, wie die Schule strukturiert ist, hat einen entscheidenden Einfluss darauf, wie die Subjekte strukturiert sind, die durch sie sozialisiert werden.

Hier knüpft die - auch von Parsons ausgehende - erweiterte Professionalisierungstheorie an (Combe/Helsper 1996; Oevermann 2009b). Kern dieser Theorie ist die Kritik an der strikt rollenförmigen und bürokratisch agierenden Schule, die einseitig auf die Erziehung und Disziplinierung der Subjekte gerichtet ist. Die professionalisierungstheoretische Betrachtung rückt die Krisenhaftigkeit von Bildungsprozessen und die Erreichung einer größtmöglichen Autonomie der Schüler_innen durch Bildungsprozesse ins Zentrum. Daraus ableitend fordert sie eine professionalisierte Schule, die sich stärker auf die Entwicklung des Potenzials der Schüler_innen konzentriert. Das bedingt ein professionalisiertes Handeln, das auf eine stellvertretende Hilfeleistung im Sinne einer ,Hilfe zur Selbsthilfe" ausgerichtet ist. Dabei wird konstatiert, dass die Schule professionalisierungsbedürftig, aber nicht professionalisiert ist (vgl. Oevermann 2002b). Fragen des Lehrberufes sollen jedoch an anderer Stelle thematisch werden. Zudem ist die Frage der Professionalisierungsbedürftigkeit und Nichtprofessionalisiertheit des Lehrberufes durchaus umstritten (zur Diskussion siehe Helsper/Tippelt 2011; Wernet 2014). Wichtig ist an dieser Stelle für das zu untersuchende Erkenntnisinteresse die Feststellung, dass die sekundäre Sozialisation in der Schule in einem Spannungsfeld angesiedelt ist. Auf der einen Seite steht die Schule als bildende Institution, die Subjekte hervorbringen soll, die das gesellschaftlich vorhandene Potenzial ausschöpfen und erweitern und die auch in der Lage sind, zukünftige Herausforderungen zu lösen. Auf der anderen Seite steht die gesell-

20 Dies gilt insbesondere für kleine Kinder, die die Schülerrolle noch nicht internalisiert haben. 
schaftliche Anforderung nach disziplinierten, rollenförmig agierenden Subjekten.

\subsubsection{Interaktion mit Artefakten und deren sozialisatorische Wirkungen}

Es bleibt, an dieser Stelle den Bogen zurückzuführen zum Gegenstand der Schulhausarchitektur. Inwiefern haben Gebäude, architektonische Artefakte oder Dinge einen Einfluss auf Bildungs- und Sozialisationsprozesse? Inwiefern haben Artefakte oder Architekturen als gebaute Umwelt einen Einfluss auf die Sozialisations-, Lern- und Bildungsprozesse?

Eine sinnaffine Konstruktion zur naturwüchsigen sozialisatorischen Interaktion zwischen Menschen findet sich bei Piagets Modell (2003) der kognitiven Entwicklung, das dieselben Strukturprinzipien auch für die Interaktion mit Dingen darlegt. Mit Piagets Lerntheorie gedacht, entstehen Erkenntnisprozesse krisenhaft dann, wenn im Interaktionsprozess zwischen Subjekt und Objekt eine Aneignung stattfindet. Das Kind ist ein aktives Wesen, das sich sequenziell entwickelt, indem es in eine Auseinandersetzung mit der Welt eintritt, diese strukturiert und dabei die Welt und sich selbst verändert. Dabei finden Erkenntnisprozesse statt, entweder indem sich das Kind an die Umwelt assimiliert: Es bringt die äußere Welt in Übereinstimmung mit der inneren Welt. Oder im Prozess der Akkomodation, indem es seine innere Welt der äußeren (Um-)Welt anpasst. Das Kind entwickelt damit durch Internalisierung der äußeren Welt neue kognitive Wahrnehmungsschemata, was gleichzeitig oft zu einer ,Anpassung ' bzw. einer Entwicklung der epistemischen Strukturen führt.

Wesentlich ist, dass Erkenntnisprozesse in einer Interaktion stattfinden. Wie Piaget ausführt, ist der „entscheidende Punkt unserer Theorie (...), dass Erkenntnis sich aus Interaktionen zwischen dem Subjekt und dem Objekt ergibt, die reichhaltiger sind als alles, was die Objekte von sich aus liefern können“ (Piaget 2003: 74, Hervorhebungen im Original). Der Grad möglicher Erkenntnis ist also nicht den physisch-materialen Eigenschaften eines Objektes immanent, sondern beruht auf den Eigenschaften von Handlungen, die mit dem Objekt vollzogen werden können.

An dieser Stelle wird auch der Gegenstand Architektur bzw. der architektursoziologische und bildungssoziologische Blick auf Schulhäuser interessant, 
insbesondere dann, wenn sie als ,pädagogische Objekte' betrachtet werden. Denn als gebaute Umwelt sprechen auch Schulhäuser eine Sprache: Sie speichern Sinn und geben soziale Realitäten und den Möglichkeitsraum von Aneignungen vor. Für die nachfolgenden Analysen ist daher die Frage bedeutsam, inwiefern Architekturen Bildungsprozesse unterstützen und damit ,pädagogische Architekturen' sind oder inwiefern sie vor allem als Routinen wirksam sind, die auf eine disziplinierende Anpassungsleistung der Sozialisanden gerichtet sind.

Ein weiterer Punkt, der in den Analysen mit betrachtet werden soll, ist, dass Kinder noch nicht autonom sind. Sie bedürfen einer Obhut, worauf auch die Architektur in irgendeiner Form reagieren muss. Dies ist in besonderem Maße dadurch gegeben, dass Schulen als Orte von Massenveranstaltungen großer Kindergruppen konzipiert sind.

Bereits angesprochen wurde, dass der primäre Aneignungsmodus von Kindern die perzeptive Wahrnehmungs-, Körper- und Leiberfahrung ist. Es ist gedankenexperimentell also anzunehmen, dass Architektur in irgendeiner Art und Weise diesen Umstand aufnehmen muss, z. B. in der Bereitstellung von Räumen (und Zeiten) des Nichtunterrichts (etwa Pausenplätzen). Dies gilt aber auch dafür, dass Schulhäuser Räume von körper- und leibgebundenen Erfahrungskonstitutionen konzipieren müssen.

Zum Auftrag der Schulhäuser gehört, dass Schulen nicht ausschließlich eine pädagogische Funktion innehaben. Gerade als öffentliche und staatliche Gebäude sind Schulhäuser Repräsentationsbauten, die die Staatlichkeit verkörpern. Dies ist für den Gebäudetyp Schule bezeichnend, da Schulen dezentral gebaut wurden und werden. Insbesondere bei der Genese des Nationalstaates waren Schulhäuser damit auch ein Mittel der staatlichen Manifestation bis in die hintersten Täler und entlegensten Dörfer. Schulbauten nehmen zusätzlich zur Repräsentationsfunktion auch andere öffentliche Funktionen wahr. Insbesondere in kleineren Gemeinden werden Schulbauten oft mit multifunktionellen Räumen konzipiert, die auch als Gemeinderäume benutzt werden können oder Vereinstätigkeiten am Abend beherbergen. Insgesamt haben Schulhäuser eine wichtige Funktion für die Generierung kommunaler Identitäten.

Diese zwangsläufig unvollständig entworfene Konturierung ist in einer zeitdiagnostischen Betrachtung zu verorten, auch wenn diese einen relativ langen Zeitraum umfasst. Im Folgenden sollen die beiden theoretischen Erörterungen zur Fragestellung verbunden werden, bevor die (schul)pädagogischen und 
sozialisatorischen Fragen von Schule direkt am empirischen Material weiterdiskutiert werden.

\subsection{Ableitung der Fragestellung}

Schulhäuser werden in einem komplexen Prozess mit Beteiligung vieler Akteure geplant und gebaut. Sie entstammen einer kollektiven Praxis. Zudem entspringen sie einem Entwurf, der eine spezifische Praxis gedankenexperimentell vorwegnimmt. Sie sind eine Manifestierung jeweils epochal verankerter Deutungsmuster über Schule, Pädagogik und Kindheit. Als dauerhafte Objektivationen verkörpern Schulbauten also ehemals in die Zukunft geplante Entwürfe. Zugleich stellen sie Kontinuität her, indem sie die in Stein gehauenen Sinnstrukturen dauerhaft verfestigen. Daher eignet sich die Architekturanalyse, weil sie verspricht, dass ,jene kulturellen Traditionen und Sinnstrukturen synchronisch und diachronisch aufgearbeitet werden, die in besonderer Weise die historische Entwicklung des Bildungssystems und der familialen Erziehungspraxis beeinflussen“ (Oevermann, 2012, S. 196). Dabei müssten laut Oevermann die

„Wissenssysteme und Deutungsmuster, die die expliziten Bildungsziele fundieren, als relativ verselbständigte objektive Argumentationsstrukturen (...) [behandelt werden], die gleichsam hinter dem Rücken der subjektiven Intentionalität der einzelnen Personen das Handeln im Sinne eines „Habitus“ (Bourdieu) bestimmen.“(ebd.)

Die Architekturanalyse verspricht diesem Umstand nahezukommen. Schulhäuser sind Entwürfe einer idealen Praxis und verkörpern damit jeweilige Ideale von ,guter Schule‘. Die Architektur entfaltet eine sublime, aber effektive Wirkung auf die Möglichkeiten der schulischen Praktiken. In der Rekonstruktion der Brüche und Anpassungsleistungen durch Um-, An- und Erweiterungsbauten können auch neue Anforderungen in der Normativität ,guter Schule' rekonstruiert werden. Dies gelingt jedoch nur dann, wenn die innere Gesetzmäßigkeit erschlossen werden kann und sich die Analyse nicht nur auf die Deutung von manifesten Wirkungen und Expressionen beschränkt.

Das übergeordnete Erkenntnisinteresse der vorliegenden Arbeit ist die Rekonstruktion der Eigenlogik von Schulbauten in ihren manifesten und latenten Sinngehalten. Dabei wird zwischen zwei zusammenhängenden Fragebereichen 
mit mehreren Unterfragen unterschieden, die sich aus den konstitutionstheoretischen Erörterungen ableiten:

Zum Ersten wird rekonstruiert, wie die Sesshaftigkeit der Schule architektonisch gedeutet und realisiert wird. Dazu gehört die Repräsentation nach außen, die Konstituierung der Innen-Außen-Abgrenzung und die Funktionalität der Schulen innerhalb des Siedlungsgefüges. Thematisiert wird also, welche Denkund Wissensformen sich in der Architektur der Schule nach außen manifestieren.

Zum Zweiten wird als eigenständiger Bereich davon abgegrenzt, wie das Handlungsproblem von Schule - also Bildung und Sozialisation - durch Architektur umgesetzt wird. Dabei interessieren die Funktionalität des Gebäudes und die Frage, welche Suggestion ein Gebäude entfaltet, um Schule zu unterstützen.

\subsubsection{Zur Sesshaftigkeit der Schule}

Mit der Sesshaftigkeit von Schule ist insbesondere die Repräsentationsfunktion der Architektur thematisch. Es wird den Fragen nachgegangen, welche Sinnstrukturen sich in der Selbstdarstellung der Schule verkörpern: Welche Regeln und Sinnbilder können als Eigensinn einer jeweiligen Schulförmigkeit realisiert werden, die den Gebäudetyp Schulhaus ausmachen und damit als ,Vorbewusstes" der Schule fungieren? Wie wird die Funktionalität der Schule als Schule nach außen repräsentiert? Und last but not least, wie wird die Bauherrschaft nach außen repräsentiert?

Mit der Frage der Repräsentation ist auch die Frage der Innen-AußenAbgrenzung angesprochen. Um diese zu fokussieren, müssen die Schulbauten innerhalb ihrer Siedlungsumgebung betrachtet werden. Daher wird die Positionalität der Schulbauten, ihre Funktionalität innerhalb des Siedlungsgefüges und die damit einhergehenden Bedeutungsstrukturen in den Fallrekonstruktionen mit thematisiert. 


\subsubsection{Zur Funktionalität schulischer Architektur}

Zum Zweiten richtet sich das Erkenntnisinteresse auf die Funktionalität der Schulhausarchitektur. Hier sind mehrere Bereiche zu differenzieren: Auf einer deskriptiven Ebene kann das Raumprogramm einer Schulanlage als explizite ,Theorie der Schule" gelesen werden. Für das Vorhaben bedeutender sind allerdings die impliziten und latenten Sinngehalte: Welche Ideen und Vorstellungen über Pädagogik, Schule, Kindheit und Sozialisation lassen sich aus der Materialität der Schulbauten rekonstruieren? Inwiefern ist Architektur oder Teile davon eine ,pädagogische Architektur', die sich explizit oder implizit auf pädagogische und/oder Sozialisationspraktiken richtet? Was kann mit der Analyse der Architektur über die Praktiken an der Institution Schule gesagt werden? Damit sind diejenigen Bereiche angesprochen, die begrifflich als "Programm des Raums“ oder als Gestik der Architektur gefasst werden.

Aus dem Raumprogramm, dem Programm der Räume und den Raum(an)ordnungen können Aussagen über die Akteure und deren Beziehungen gemacht werden: Wie werden die Beziehungen und Binnenbeziehungen zwischen Lehrpersonen, Schüler_innen und anderen Akteuren gedacht und präformiert? Welche Praktiken werden durch die Architektur unterstützt, und welche werden eher unterbunden?

Da Schulhäuser beständig sind, liegt ein weiterer Fokus des Erkenntnisinteresses auf der Genese des Bildungswesens und den (Um-)Brüchen zwischen der räumlich-architektonischen Konstitution von Schule und den zeitgebundenen und sich ändernden pädagogischen Praktiken. Architektur als Datenmaterial und Forschungsgegenstand verspricht Ergebnisse über besonders beständige Strukturen, die gewollt oder unbewusst die Schule durch die Architektur in ihrer heutigen Ausdrucksmaterialität und Bedeutungsstruktur präformieren.

Nach diesen Herleitungen und der Konturierung der Fragestellung werden im nächsten Kapitel der methodische Umgang mit Architektur als Datenmaterial geklärt. 
Open Access Dieses Kapitel wird unter der Creative Commons Namensnennung 4.0 International Lizenz (http://creativecommons.org/licenses/by/4.0/deed.de) veröffentlicht, welche die Nutzung, Vervielfältigung, Bearbeitung, Verbreitung und Wiedergabe in jeglichem Medium und Format erlaubt, sofern Sie den/die ursprünglichen Autor(en) und die Quelle ordnungsgemäß nennen, einen Link zur Creative Commons Lizenz beifügen und angeben, ob Änderungen vorgenommen wurden.

Die in diesem Kapitel enthaltenen Bilder und sonstiges Drittmaterial unterliegen ebenfalls der genannten Creative Commons Lizenz, sofern sich aus der Abbildungslegende nichts anderes ergibt. Sofern das betreffende Material nicht unter der genannten Creative Commons Lizenz steht und die betreffende Handlung nicht nach gesetzlichen Vorschriften erlaubt ist, ist für die oben aufgeführten Weiterverwendungen des Materials die Einwilligung des jeweiligen Rechteinhabers einzuholen.

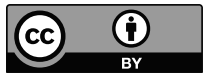

